\title{
HCG 31: a multiple merger in progress ${ }^{\star} \star \star \star$
}

\author{
P. Amram ${ }^{1}$, C. Mendes de Oliveira ${ }^{2}$, H. Plana ${ }^{3}$, C. Balkowski ${ }^{4}$, and O. Hernandez ${ }^{5,1}$ \\ ${ }^{1}$ Laboratoire d'Astrophysique de Marseille, Observatoire Astronomique Marseille-Provence, Université de Provence \& CNRS, \\ 2 Place Le Verrier, 13248 Marseille Cedex 4, France \\ 2 Departamento de Astronomia, Instituto de Astronomia, Geofísica e Ciências Atmosféricas da USP, Rua do Matão 1226, \\ Cidade Universitária, 05508-090 São Paulo, Brazil \\ ${ }^{3}$ Laboratório de Astrofísica Teórica e Observacional, Universidade Estadual de Santa Cruz, Ilhéus, BA, Brazil \\ ${ }^{4}$ Observatoire de Paris, GEPI, CNRS and Université Paris 7, 5 Place Jules Janssen, 92195 Meudon Cedex, France \\ ${ }^{5}$ Laboratoire d'Astrophysique Expérimentale, Département de Physique de l'Université de Montréal and Observatoire du \\ Mont-Mégantic, CP 6128, Succ. centre-ville, Montréal, Qc, H3C 3J7, Canada
}

Received 18 August 2005 / Accepted 19 April 2007

\section{ABSTRACT}

Context. HCG 31 is one of the most intriguing compact groups in Hickson's catalogue. It contains a central pair of interacting dwarf galaxies and other small members that are highly peculiar.

Aims. The main goal of this study is to investigate the evolutionary stage of the group and measure the internal kinematics of the individual galaxies and of the possible tidal dwarf galaxies.

Methods. We obtained new Fabry-Perot data cubes, including $\mathrm{H} \alpha$ images, velocity fields, velocity dispersion maps, lambda maps, position-velocity plots and rotation curves. We have taken advantage of the high spatial (sampling of $\sim 0.4$ arcsec) and spectral (velocity sampling of $\sim 3 \mathrm{~km} \mathrm{~s}^{-1}$ ) resolutions of our Fabry-Perot data.

Results. Kinematic peculiarities and overlapping double velocity components are measurable throughout the velocity field of $\mathrm{A}+\mathrm{C}$, with no signature of either distinct rotating disks or a single rotating galaxy. The velocity dispersion map shows widespread lowvelocity values throughout the group (consistent with the turbulent velocity of the gas), except in a narrow interface between A and C, where the merging may be occurring. The velocity curves of four candidate tidal dwarf galaxies (regions E, F, A1, and A2) show flat velocity patterns in two cases (A1 and F) and gradients with amplitudes of $A \sim 30-40 \mathrm{~km} \mathrm{~s}^{-1}$ in two others (E and A2). Our measurements show good agreement with previous optical literature data, but our data set has a much improved velocity sampling and deeper coverage. Moreover, within the star-forming regions of the group, our data profit from the higher spatial resolution with respect to HI data.

Conclusions. The dynamics of the $\mathrm{A}+\mathrm{C}$ system, with two main velocity components of approximately the same intensities, indicate that it is in a pre-merger stage. The two disks, in a bound orbit and in the process of merging, have had at least one earlier passage. They rotate with almost parallel spin axes like a set of gear wheels. This prograde encounter and the high star-formation rates favor the formation of a new disk. Object F may turn into a tidal dwarf galaxy bound to the group.

Key words. galaxies: general - galaxies: kinematics and dynamics - galaxies: evolution - galaxies: interactions - galaxies: ISM galaxies: formation

\section{Introduction}

Two-dimensional kinematic data from the Fabry-Perot studies of compact groups have provided evidence that a number of galaxies in these groups have experienced frequent and strong interactions (Amram et al. 2003; Plana et al. 2003). The main findings are that: 1) group members often display dynamical peculiarities; 2) there is evidence of accretion or merging among group members for $40 \%$ of the groups; and 3) several candidate tidal dwarf galaxies (TDGs) are identified within compact groups. Despite the observed interacting features, however, the TullyFisher relation of compact group galaxies is similar in slope and zero point to that for galaxies in less dense environments (Mendes de Oliveira et al. 2003).

HCG 31 is a compact group in which all galaxy members show signs of recent or ongoing interactions (e.g.

« Based on observations collected at the European Southern Observatory, La Silla, Chile.

$\star \star$ Appendix A is only available in electronic form at http://www . aanda.org
Mendes de Oliveira \& Hickson 1994) and TDGs have been identified in it (Mendes de Oliveira et al. 2006). Objects A, B, C, D were first identified by Hickon (1982). Sulentic (1987) has reconfigured this group by rejecting the faint " $\mathrm{D}$ " component and by adding the component lately named " $F$ " by Rubin et al. (1991). Finally, Rubin et al. (1991) add two more components "E", "G" and "Q" to the configuration given by Sulentic (1987). This is not surprising given that it has a galaxy-galaxy median projected separation of only $22 \mathrm{kpc}$ and a radial velocity dispersion of $57 \mathrm{~km} \mathrm{~s}^{-1}$ (computed from the new values listed in Table 2 of Mendes de Oliveira et al. 2006). This group was not included in the Tully-Fisher study of Mendes de Oliveira et al. (2003) because the rotation curves of its members were too peculiar.

Several recent works have been specially dedicated to the internal kinematics of this group and its members: Richer et al. (2003) published Fabry-Perot observations and multi-object spectroscopy, López-Sánchez et al. (2004) presented long-slit spectroscopy and broad-band optical and near-infrared images, and Verdes-Montenegro et al. (2005) studied the HI content and 
kinematics of the group as a whole. The HI gas in HCG 31 is found within the galaxies and in the tidal tails, in contrast with HCG 92 (Stephan's Quintet), a more evolved group for which the gas is found only in the intra-group medium (Williams et al. 2002). Three scenarios have been put forward to explain the nature of the central system $\mathrm{A}+\mathrm{C}$ : (i) these are two systems that are in a pre-merger phase (e.g. Amram et al. 2004; Verdes-Montenegro et al. 2005); (ii) the system is a late-stage merger (e.g. Williams et al. 1991); or (iii) it is a single interacting galaxy (Richer et al. 2003). Unrevealing the history of this group is clearly a challenge that should be undertaken from the observational and modeling sides.

In this paper we add to this scenery our Fabry-Perot observations of the internal kinematics of several of the group members. Analysis of part of these data was done in Amram et al. (2004). Our data comprise $\mathrm{H} \alpha$ velocity maps and monochromatic images of the central region of the group, which includes objects $\mathrm{A}+\mathrm{C}, \mathrm{B}, \mathrm{E}, \mathrm{F}, \mathrm{G}$, and $\mathrm{H}$ (identified in Fig. 1, except $\mathrm{G}$, which is outside the field). The diameter of the group as identified by Hickson (1982) subtends a diameter of 1 arcmin $(\sim 16 \mathrm{kpc})$ and has to be increased by a factor $\sim 2$ if galaxy $\mathrm{G}$ is included.

In Sect. 2, we describe our Fabry-Perot observations, the observational techniques, and data reduction analysis. In Sect. 3, we present the results on the velocity fields, the rotation curves (RCs), and velocity dispersion maps. In Sect. 4 we compare our results with those presented in severalof the previous kinematic studies cited above. In Sect. 5, we discuss of the results. Finally, in Sect. 6 we give the summary and conclusions. In Appendix A, we give a detailed comparison of our data set with previous optical and HI data sets.

A Hubble constant $H_{0}=75 \mathrm{~km} \mathrm{~s}^{-1} \mathrm{Mpc}^{-1}$ is used throughout this paper. We adopt a distance to the group of $54.8 \mathrm{Mpc}$, obtained from the redshift of the group and the Hubble law, and hence 1 arcsec $\sim 0.27 \mathrm{kpc}$.

\section{Observations and data reduction}

Observations were carried out in two runs at the European Southern Observatory $3.6 \mathrm{~m}$ telescope (ESO $3.6 \mathrm{~m}$ ) in August 1995 and in August 2000. Table 1 contains the journal of the observations and observational setups for both runs. A Fabry-Perot instrument with an interference order of $p=796$ and a Finesse of $F=12$ was used for the 1995 data set. Analysis of this first data set suggested that multiple gaseous components were present in different regions of the group: line broadening was seen in the central regions of galaxies HCG $31 \mathrm{~A}$ and C and double peaks were visible in the overlapping regions between these two galaxies. Nevertheless, the spectral resolution of $R=9400$ was not high enough to disentangle the components of the broad line and to separate the multiple peaks unambiguously. The data taken in August 2000 used a higher-order ( $p=1938)$ Fabry-Perot scanning interferometer with a much better Finesse, $F=24$, giving a spectral resolution of $R=45900$. During both runs, the Fabry-Perot instrument CIGALE (Amram et al. 1991; Gach et al. 2002) ${ }^{1}$ was used. It is composed of a focal reducer (bringing the original $\mathrm{f} / 8$ focal ratio of the Cassegrain focus to $\mathrm{f} / 2$ ), an interference filter, a scanning Fabry-Perot and a new generation (GaAs tube) of the Image Photon Counting

1 The instrument CIGALE (for CInématique des GALaxieEs) is a visiting instrument from the "Laboratoire d'Astrophysique de Marseille",

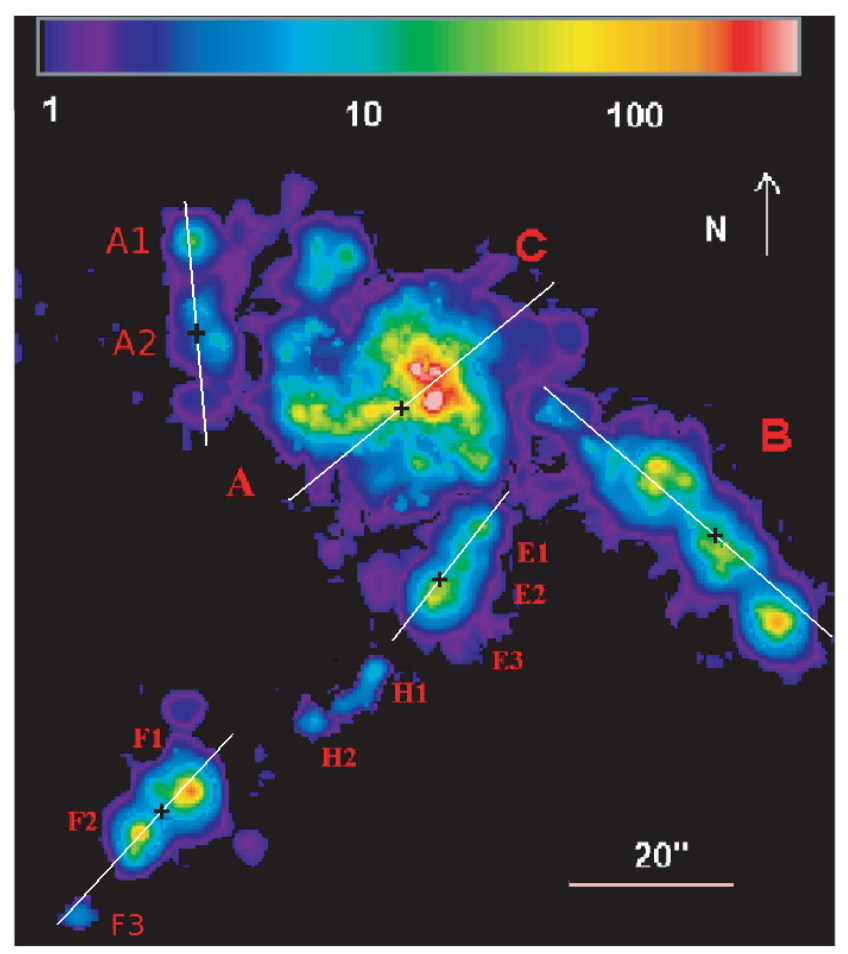

Fig. 1. Emission-line map of HCG 31 from the high-resolution data set using a logarithmic scale and a variable spatial smoothing, from full resolution $(\sim 0.4$ arcsec $)$ in the central regions to a Gaussian beam of 3.6 arcsec in the outskirts (see text for more details). The brightest knot at the center of $\mathrm{A}+\mathrm{C}$ is 700 times more intense than the lowest level intensity, at $3 \sigma$ above the background. The white lines represent the slit orientations used to obtain the rotation curves and the velocity plots given in Fig. 9. The black crosses represent the position of the centers used to build the rotation curves given in the same figure.

System (IPCS) ${ }^{2}$. An IPCS was used instead of a CCD to minimize seeing variation, transparency and sky foreground fluctuations. With a time sampling of 1/50 s and zero readout noise, the use of this GaAs detector made it possible to scan the interferometer rapidly (typically 5 to $15 \mathrm{~s}$ per channel), avoiding sky transparency, air-mass, and seeing variation problems during the exposures and thus has several advantages over a CCD, for this specific application (Gach et al. 2002).

Reduction of the data cubes were performed using the CIGALE/ADHOCw software (Boulesteix 2002). The data reduction procedure has been described in Amram et al. (1992). Wavelength calibration was obtained by scanning the narrow Ne $6599 \AA$ line under the same conditions as the observations. Velocities measured relative to the systemic velocity are very accurate, with an error of a fraction of a channel width $\left(<3 \mathrm{~km} \mathrm{~s}^{-1}\right)$ over the whole field. For our adopted distance to the group of $54.8 \mathrm{Mpc}, 1$ pixel corresponds to $\sim 0.24(0.12) \mathrm{kpc}$ for the 1995 (2000) data set.

The data presented in this paper are both from the 1995 and 2000 observing runs. Hereafter, we refer to the 1995 lowresolution data as the LR data set and to the 2000 high-resolution data as the HR data set. The LR data set goes deeper and is used to emphasize the connections between the different objects of HCG 31; the LR data set is also used to perform the corrections due to a possible free spectral-range jump in the HR data set.

2 The IPCS and the interference filters used are from the "Laboratoire d'Astrophysique Expérimentale", Université de Montréal. 
Table 1. Journal of Fabry-Perot observations.

\begin{tabular}{|c|c|c|c|}
\hline & & $\overline{\overline{\text { Run } 1}}$ & $\overline{\overline{R u n} 2}$ \\
\hline Telescope & & \multicolumn{2}{|c|}{ ESO $3.6 \mathrm{~m}$} \\
\hline Equipment & & \multicolumn{2}{|c|}{ CIGALE@ Cassegrain } \\
\hline Calibration & Neon Comparison light & \multicolumn{2}{|c|}{$6598.95 \AA$} \\
\hline \multirow[t]{3}{*}{ Interference filter } & Central wavelength & \multicolumn{2}{|c|}{$6651 \AA$} \\
\hline & FWHM & \multicolumn{2}{|c|}{$20 \AA$} \\
\hline & Transmission at maximum & \multicolumn{2}{|c|}{0.7} \\
\hline Date & & 1995, August, 28 & 2000, September, 28 \\
\hline Exposure time & Total & $105 \mathrm{~min}$ & $72 \mathrm{~min}$ \\
\hline Seeing & & $\sim 1 \operatorname{arcsec}$ & $\sim 0.7 \operatorname{arcsec}$ \\
\hline Detector & IPCS & Thomson tube & GaAs tube \\
\hline \multirow[t]{4}{*}{ Fabry-Perot } & Interference Order & $796 @ 6562.78 \AA$ & $1938 @ 6562.78 \AA$ \\
\hline & Free Spectral Range at $\mathrm{H} \alpha$ & $380 \mathrm{~km} \mathrm{~s}^{-1}$ & $155 \mathrm{~km} \mathrm{~s}^{-1}$ \\
\hline & Finesse $^{(1)}$ at $\mathrm{H} \alpha$ & 12 & 24 \\
\hline & Spectral resolution at $\mathrm{H} \alpha$ & $9400^{(2)}$ & $45900^{(2)}$ \\
\hline \multirow[t]{4}{*}{ Sampling } & Number of Scanning Steps & 24 & 48 \\
\hline & Sampling Step & $0.35 \AA\left(16 \mathrm{~km} \mathrm{~s}^{-1}\right)$ & $7.24 \times 10^{-2} \AA\left(3.2 \mathrm{~km} \mathrm{~s}^{-1}\right)$ \\
\hline & Total Field & $170 \times 170 \operatorname{arcsec}^{2}\left(256 \times 256 \mathrm{px}^{2}\right)$ & $207 \times 207 \operatorname{arcsec}^{2}\left(512 \times 512{\left.p x^{2}\right)}^{2}\right.$ \\
\hline & Pixel Size & $0.910 \operatorname{arcsec}(\sim 0.24 \mathrm{kpc})$ & $0.405 \operatorname{arcsec}^{(3)}(\sim 0.12 \mathrm{kpc})$ \\
\hline
\end{tabular}

(1) Mean Finesse through the field of view.

(2) For a signal to noise ratio of 5 at the sample step.

(3) After binning $2 \times 2$, the original GaAs system providing $1024 \times 1024 \mathrm{px}^{2}$.

The HR data set is used to trace the kinematics of the galaxies and regions of the group.

\section{Results}

\subsection{Ho maps}

Figure 1 shows the emission line map of the central region of HCG 31, with the identifications of the various observed regions (data from the HR data set). The white segments and black crosses overplotted onto this figure are later explained in the caption of Fig. 9.

Our Fabry-Perot $\mathrm{H} \alpha$ images, which do not contain [NII] emission lines, are very similar to the narrow band filter $\mathrm{H} \alpha+[\mathrm{NII}]$ images obtained by Iglesias-Páramo \& Vílchez (1997) and by Iglesias-Páramo \& Vílchez (2001) in terms of detailed morphology, intensity of the knots, seeing conditions and detection limit. In order to emphasize the brightest and the faintest $\mathrm{H} \alpha$ structures of the group, we have performed an adaptive binning shown in Fig. 1 using a logarithmic scale. The spatial adaptive binning consists of using a beam adapted to the signal-to-noise ratio, ranging from 0.4 arcsec in the regions where the signal-to-noise ratio is high, up to $3.6 \mathrm{arcsec}$ in the faintest areas.

Galaxy $\mathrm{A}+\mathrm{C}$ shows nuclear $\mathrm{H} \alpha$ emission and several resolved HII regions. In $\mathrm{H} \alpha$, the brighter component of the HCG $31 \mathrm{C}$ nucleus is 10 times the intensity of the nucleus of A. The bright $\mathrm{H} \alpha$ knots, as well as the strongest red continuum levels, are located on the overlapping $\mathrm{A}+\mathrm{C}$ region, indicating the main starburst region.

Galaxy B presents a maximum in the red continuum emission located at its center. It has a relatively normal blue disk and shows three main bursts of star formation. The one to the NE is resolved in some smaller knots and the nuclear knot is weaker in $\mathrm{H} \alpha$ than the disk ones. A small chain of faint starbursts links galaxy B with the pair $\mathrm{A}+\mathrm{C}$.

\subsection{Ho profiles}

In Figs. 2 and 3 we display a few examples of $\mathrm{H} \alpha$ profiles throughout different areas of the group. The $\mathrm{H} \alpha$ images and profiles obtained during both runs are shown (different beams and scales - linear/logarithmic - are used, see the figure captions for details). All the data (isocontours and $\mathrm{H} \alpha$ profiles) shown in Fig. 2 come from the LR data set. All the data (isocontours and $\mathrm{H} \alpha$ profiles) shown in Fig. 3 come from the HR data set.

\subsubsection{Low-resolution $\mathrm{H} \alpha$ profiles}

The profiles displayed in Fig. 2 (LR data set) from $\mathrm{c}$ to $\mathrm{f}$ show a complex structure: asymmetric shapes are seen, but they are due to the multiple components that cannot be resolved (as can be confirmed in Fig. 3, with the HR data set).

At low resolution, the southern tidal arm is traced fairly well by $\mathrm{H} \alpha$ emission from galaxies $\mathrm{A}+\mathrm{C}$ to object $\mathrm{F}$ (see the bottom area of Figs. 2c and e. In particular, broad and asymmetric profiles are observed inside region $\mathrm{H}$ (Fig. 2f). This could indicate complex streaming motions within the tidal arm where the gas density is not high enough to produce a homogenous starburst like the one observed in galaxy F. Indeed, in galaxy $\mathrm{F}$ the profiles are single Gaussians (Fig. 2f). The flux intensity in region $\mathrm{H}$ is $\sim 100$ times fainter than that of knot $\mathrm{F}^{3}$. To emphasize the fainter profiles, we have normalized all the profile heights to the maximum of each small box in panels $d$ and $f$. The intensity in each component in region $\mathrm{H}$ has, on average, a level of $\sim 3 \sigma$ above sky (the latter is measured from other regions of same size, free of any galactic emission). It is clear then that regions $\mathrm{E}$ and $\mathrm{H}$ are connected, because their profiles present a progressive overlap across the field. Between regions $\mathrm{H}$ and $\mathrm{F}$, however, there is a difference in velocity of $\sim 100 \mathrm{~km} \mathrm{~s}^{-1}$. The double-component profiles in this region seen in Fig. $2 \mathrm{f}$ are below the $3 \sigma$ background level. They are most probably due to residuals of night sky-line subtraction.

\footnotetext{
3 due to the contrast chosen to display this figure, one cannot see the emission lines between knots E3 and F2 in Fig. 2 e.
} 


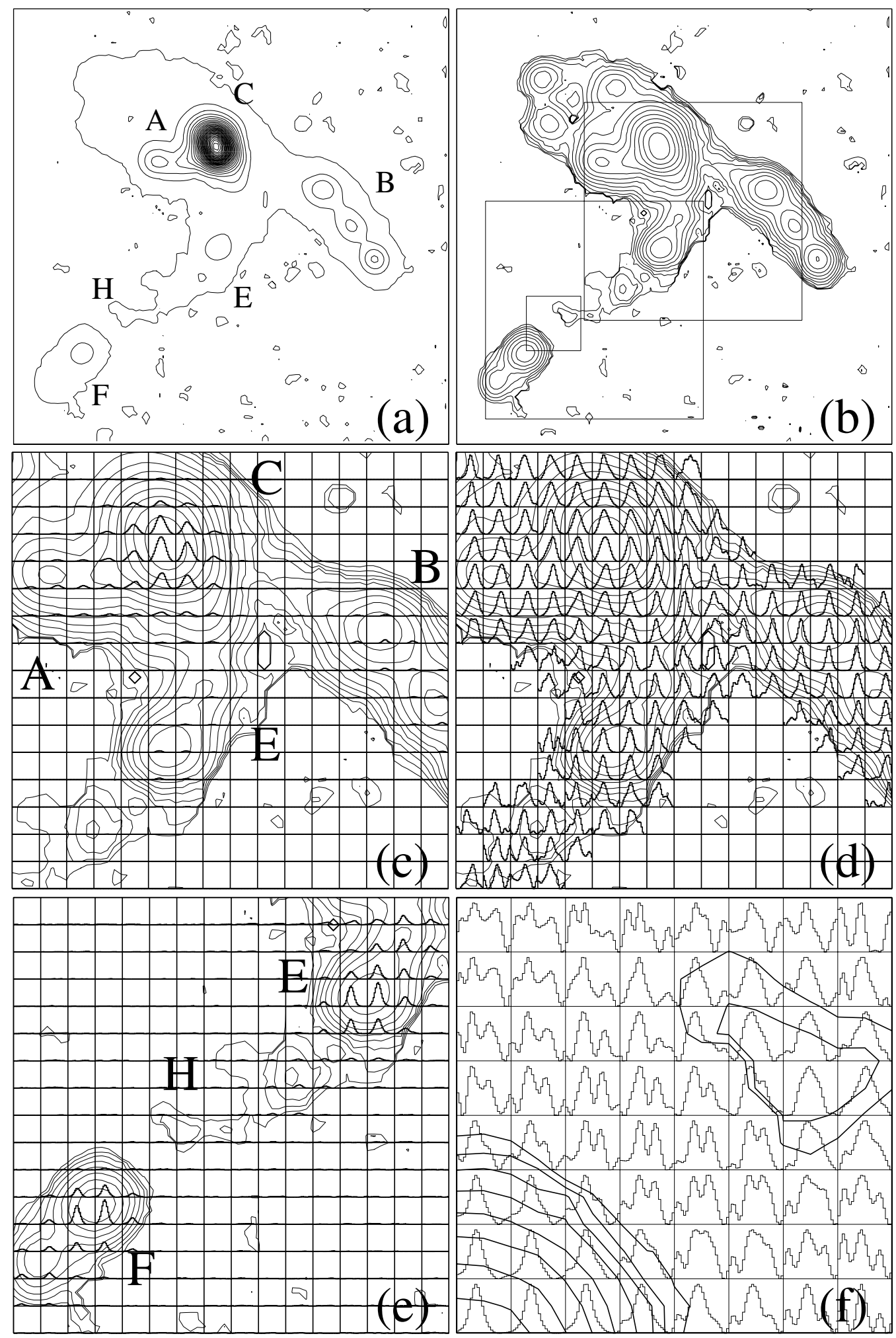

Fig. 2. $\mathrm{H} \alpha$ emission-line and isocontours from the low-resolution data set. A Gaussian beam of $\sigma=4.5$ arcsec was used for smoothing the data. The lowest contours are at a $3 \sigma$ level above the background. Panel a) monochromatic image using a linear scale. Panel b) same image using a logarithmic scale. The size of panels $a$ and $b$ is $\sim 104 \times 104 \operatorname{arcsec}^{2}$. The three squares in panel $b$ indicate the three regions zoomed in the next four panel c, $\mathrm{d}$, e, and $\mathrm{f}$ : the uppermost square of panel $\mathrm{b}$ (52 $\operatorname{arcsec}$ on a side) is zoomed in panels $\mathrm{c}$ and $\mathrm{d}$, while the large bottommost square is zoomed in e and the smallest square in $\mathrm{f}\left(7 \mathrm{arcsec}\right.$ on side). The $x$-axes of the individual small boxes represent a range of $380 \mathrm{~km} \mathrm{~s}^{-1}$. Panels c) and d): the individual small boxes represent $8 \times 8$ pixels $^{2}\left(\sim 6.5 \times 6.5 \operatorname{arcsec}^{2}\right)$. Only the brightest profiles are seen in the central regions of A+C (panel c); in order to show the faintest profiles, they have been normalized to the size of the box in panel d. Panel e) The individual small boxes represent $8 \times 8$ pixels $^{2}\left(\sim 6.5 \times 6.5 \operatorname{arcsec}^{2}\right)$. Only the brightest profiles are seen in regions $\mathrm{E}$ and $\mathrm{F}$. Panel f): the individual small boxes represent $2 \times 2$ pixels $^{2}\left(\sim 1.8 \times 1.8 \operatorname{arcsec}^{2}\right)$. In order to show the faintest profiles, they have been normalized to the size of the box in panel $\mathrm{f}$. 

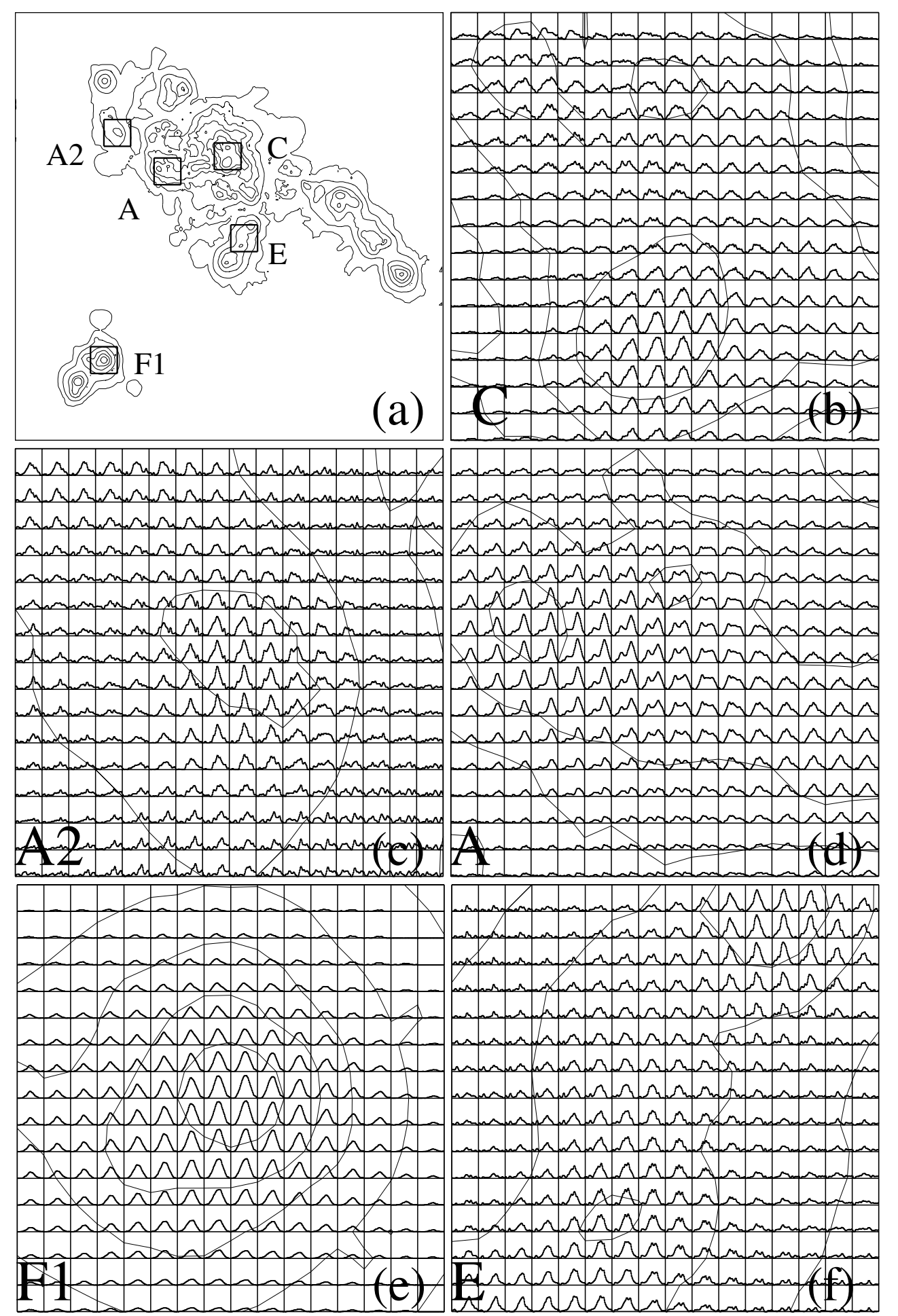

Fig. 3. $\mathrm{H} \alpha$ emission-line and isocontours from the high-resolution data set. A Gaussian beam of $\sigma \sim 1.2$ arcsec was used for smoothing the data. The lowest contours are at a $3 \sigma$ level above the background. Panel a): monochromatic image using a logarithmic scale. The size of the panel a is $\sim 104 \times 104 \operatorname{arcsec}^{2}$. Panels b) to f): emission-line contours superimposed onto the $\mathrm{H} \alpha$ profiles inside the five squares enlarged from panel a, each of size $\sim 6.5 \times 6.5 \operatorname{arcsec}^{2}$, centered on regions $\mathrm{C}$ (panel b), A2 c, A d, F1 e, and E f. The individual small boxes represent single pixels $\left(\sim 0.4 \times 0.4 \operatorname{arcsec}^{2}\right)$ on the sky. The $x$-axis (velocity) represents the free spectral range of $155 \mathrm{~km} \mathrm{~s}^{-1}$. The range and the origin of the velocities in each small box are identical. The intensities of the profiles have been normalized to the brightest profile within each panel.

\subsubsection{High-resolution $\mathrm{H} \alpha$ profiles}

The center of the $\mathrm{C}$ system is shown in Fig. $3 \mathrm{~b}$, region $\mathrm{A} 2$ is shown in Fig. 3c, the eastern extension of galaxy A in Fig. 3d, the highest intensity knot of galaxy F (noted F1) in Fig. 3e, and the base of the SW tidal arm (object E) in Fig. 3f.
At high resolution, the profiles present a complex shape almost everywhere within galaxies $\mathrm{A}, \mathrm{C}$, and $\mathrm{E}$ (see regions $\mathrm{C}, \mathrm{A} 2$, A, and E), respectively shown in Figs. 3b, c, d, f; the profiles are modeled fairly well by a double Gaussian function (Fig. 4, upper panel). In contrast, no double components are found within 

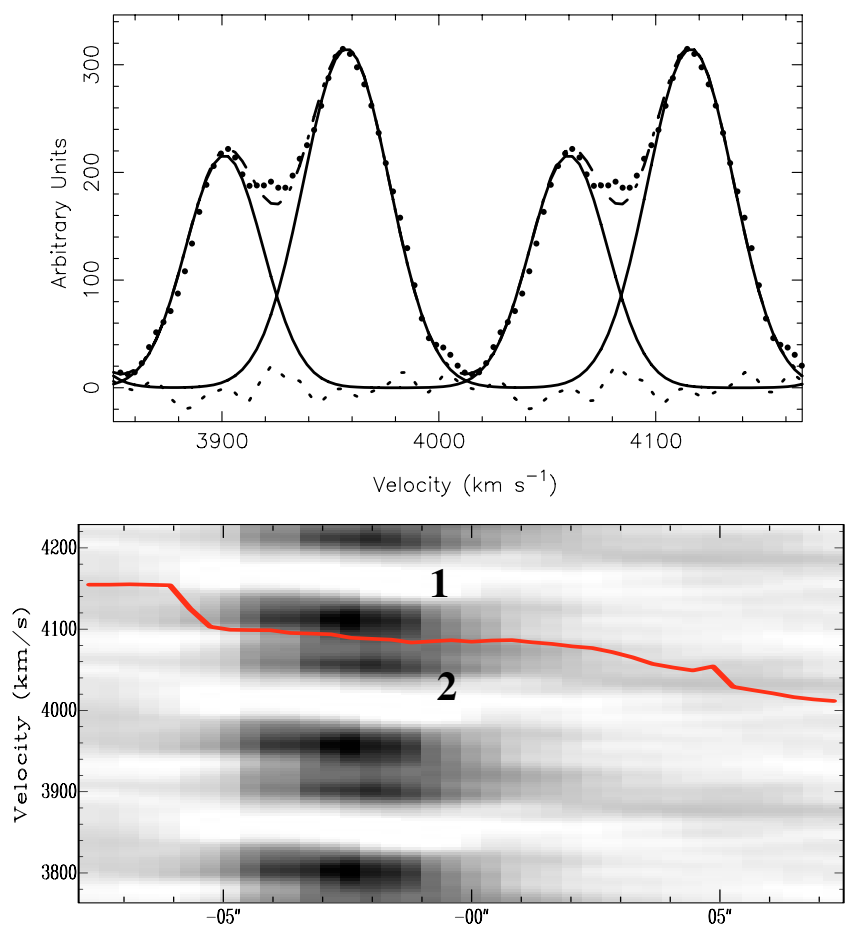

Angular Offset

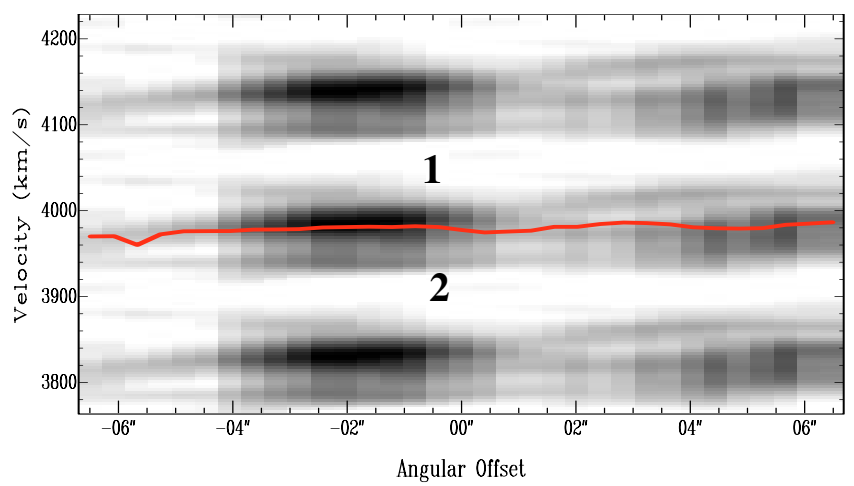

Fig. 4. (Top) Example of decomposition of an observed profile (dots) by two Gaussian components (continuous lines). The long-dashed line is the sum of the two Gaussian components and the short-dashed line is the residual. The Gaussian has been displayed twice to visualize the free spectral range recovering $\left(155 \mathrm{~km} \mathrm{~s}^{-1}\right)$. The separation between the two Gaussian components is $53 \pm(n \times) 155 \mathrm{~km} \mathrm{~s}^{-1}$ or alternatively $103 \pm(n \times) 155 \mathrm{~km} \mathrm{~s}^{-1}$, where $n$ is an integer. The velocity dispersion of the lowest and highest components are respectively 14.5 and $16.5 \mathrm{~km} \mathrm{~s}^{-1}$. (Middle and bottom) Position-velocity diagrams through galaxies $\mathrm{A}+\mathrm{C}$, the location of the two cuts are given by the two pink segments displayed in Fig. 6 (on the East, PA $=10^{\circ}$; on the West, $\left.\mathrm{PA}=170^{\circ}\right)$. For clarity, the FSR has been reproduced 3 times ( $y$-axis). The $\mathrm{H} \alpha$ flux is given in a logarithmic scale. The two different velocity components along the line-of-sight are labeled by "1" and "2". The red continuous lines represent the mean velocity extracted from the velocity field, computed from the weighted intensity barycenter of both components.

galaxy B and object F1. These regions could be modeled by single Gaussian functions (see Fig. 3e). The multiple components are resolved in several cases, see Figs. 3b-d, and Fig. 3f. In particular, the physical connection between galaxies $\mathrm{A}+\mathrm{C}$ and $\mathrm{B}$ is clearly indicated by the presence of double components. Indeed, the connection is probably real and not just a chance alignment; otherwise, the background component would be strongly

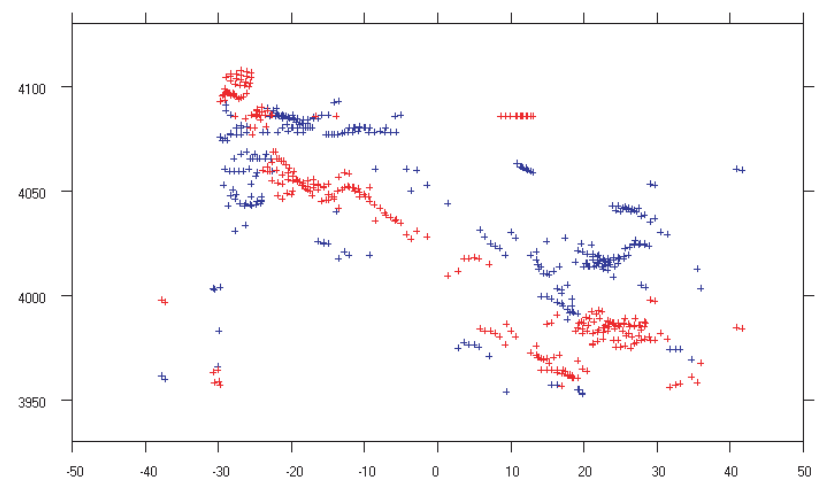

Fig. 5. Velocity components along the major axis of the $\mathrm{A}+\mathrm{C}$ complex (130 degrees). The red symbols represent the most intense component and the blue symbols the lowest intensity one.

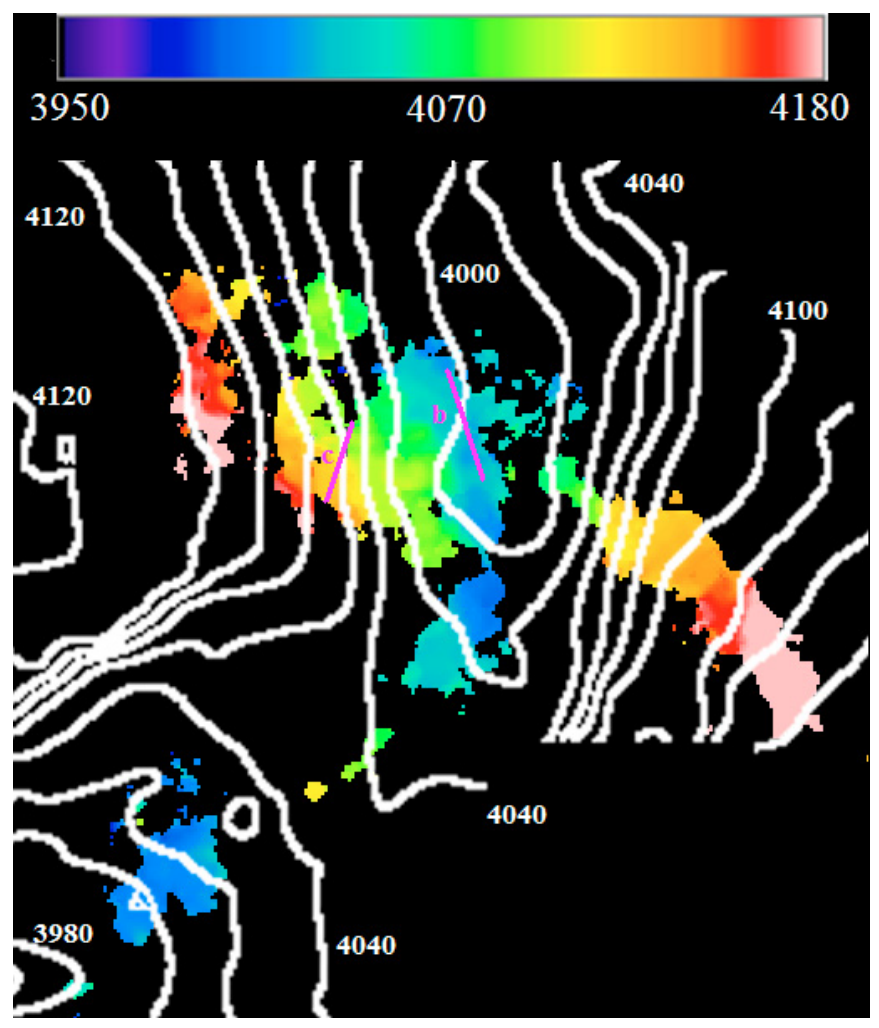

Fig. 6. $\mathrm{H} \alpha$ color velocity field of HCG 31 obtained with a $(1.2 \text { arcsec })^{2}$ Gaussian beam and a spectral sampling of $5 \mathrm{~km} \mathrm{~s}^{-1}$. The color scale is labeled in $\mathrm{km} \mathrm{s}^{-1}$. The white isovelocities and labels represent the HI component taken from Verdes-Montenegro et al. (2005). The two pink segments ( $\mathrm{PA}=10$ and $70^{\circ}$, from East to West, respectively) represent the two cuts displayed in the position-velocity diagram (see Fig. 4 middle and bottom).

affected by dust extinction due to the foreground component, which is obviously not the case.

Figure 4 (upper panel) gives an example of decomposition of an observed profile into two components for the HR case. The finesse of the etalon being high, the full-width of half maximum (FWHM) of the point-spread function (Airy function convolved with the defect function) is small with respect to the FWHM of the line; furthermore, this figure shows that sums of Gaussian components yield a suitable representation of the profiles. The separation in velocity between the different components is only known within the interval of one free-spectral range (FSR) of the interferometer $\left(155 \mathrm{~km} \mathrm{~s}^{-1}\right.$ for Fig. 4). The two sets of 


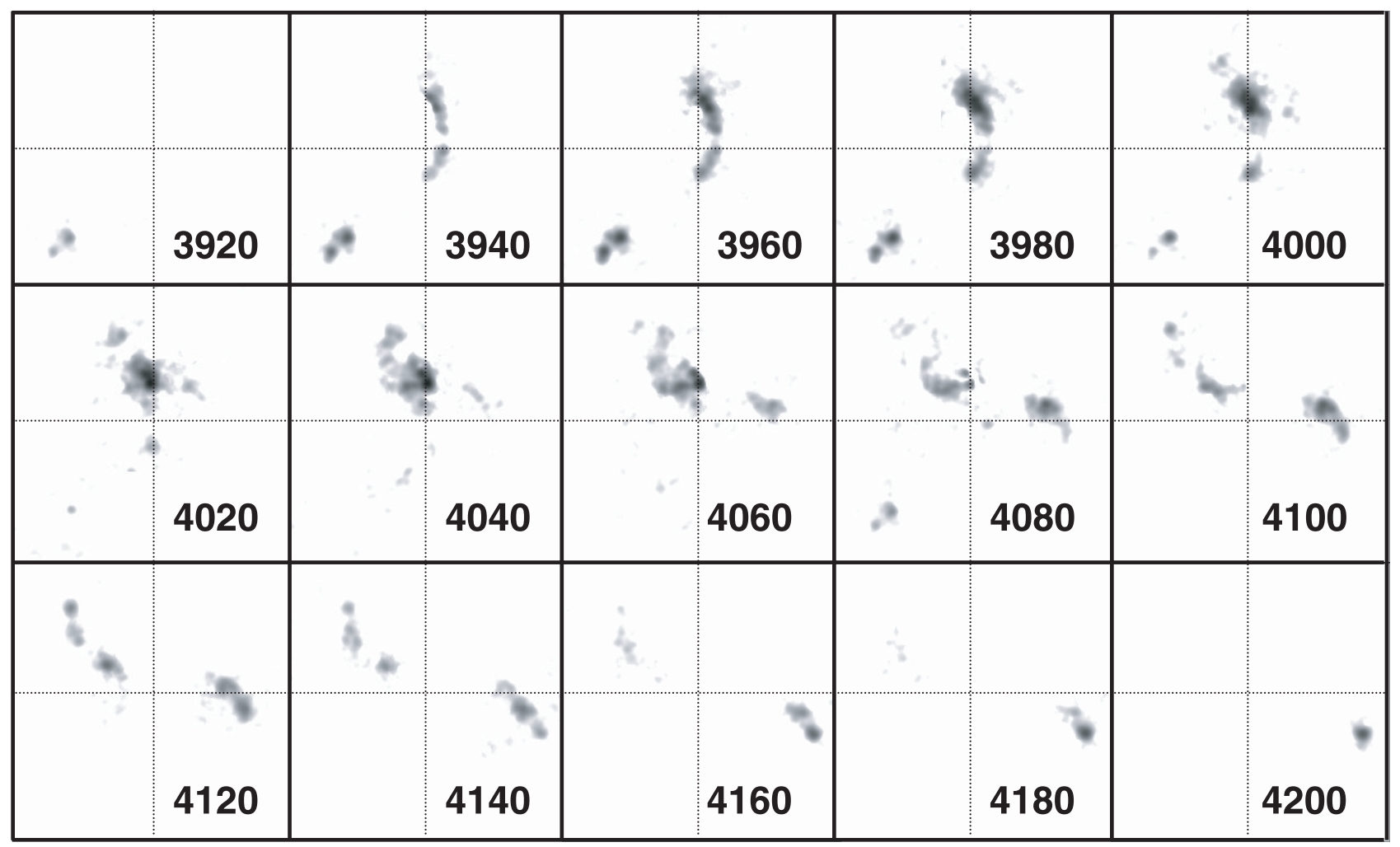

Fig. 7. A few selected lambda maps of HCG 31 . They represent the $\mathrm{H} \alpha$ emission free from the stellar continuum through 15 frames, ranging from $3920 \mathrm{~km} \mathrm{~s}^{-1}$ to $4200 \mathrm{~km} \mathrm{~s}^{-1}$. The grey scale intensity displays the logarithm of the $\mathrm{H} \alpha$ intensity levels. Each frame, oriented NE, represents the same field as the monochromatic image (Fig. 1) and velocity field (Fig. 6), i.e. $104 \times 104 \operatorname{arcsec}^{2}$. The dotted lines, separating each frame in four quadrants is intended to help reading the plots.

observations done with different FSR and the HI velocity field (Verdes-Montenegro et al. 2005) allow this velocity ambiguity take disentangled. Figure 4 (middle panel) and (bottom panel) illustrate the two velocity components (indicated by labels 1 and 2) shown in position-velocity diagrams for two different directions along $\mathrm{A}+\mathrm{C}$, as shown in Fig. 6 by the pink segments (see captions of Fig. 4 and Fig. 6). The red lines represent the intensity weighted average of both components.

In Fig. 5, the difference in velocity between the two main kinematic components in the overlapping region of $\mathrm{A}+\mathrm{C}$ is plotted. The purpose of this figure is to check whether the double profiles in $\mathrm{A}+\mathrm{C}$ are due to star formation in one single galaxy (as suggested by Richer et al. 2003) or merging of two systems followed by star formation. It can be seen that the velocity range is wider for the highest-intensity component. If the second component were only due to expanding bubbles in star-forming regions, due to the large number of HII regions, their small sizes, and added to projection effects, all values of velocity differences would be expected and not fixed values as observed. The fairly fixed velocity differences between the two components strongly suggest that the double velocity components in the interface of $\mathrm{A}+\mathrm{C}$ come from the merger of two separate entities and not from star formation in one single galaxy.

\subsection{Velocity fields, lambda maps, and velocity dispersion map}

Figure 6 shows the highest-resolution Fabry-Perot map (HR data set), displaying the velocity field of the central region of HCG 31. The HI velocity contours of Verdes-Montenegro et al. (2005) are superposed onto it. The two

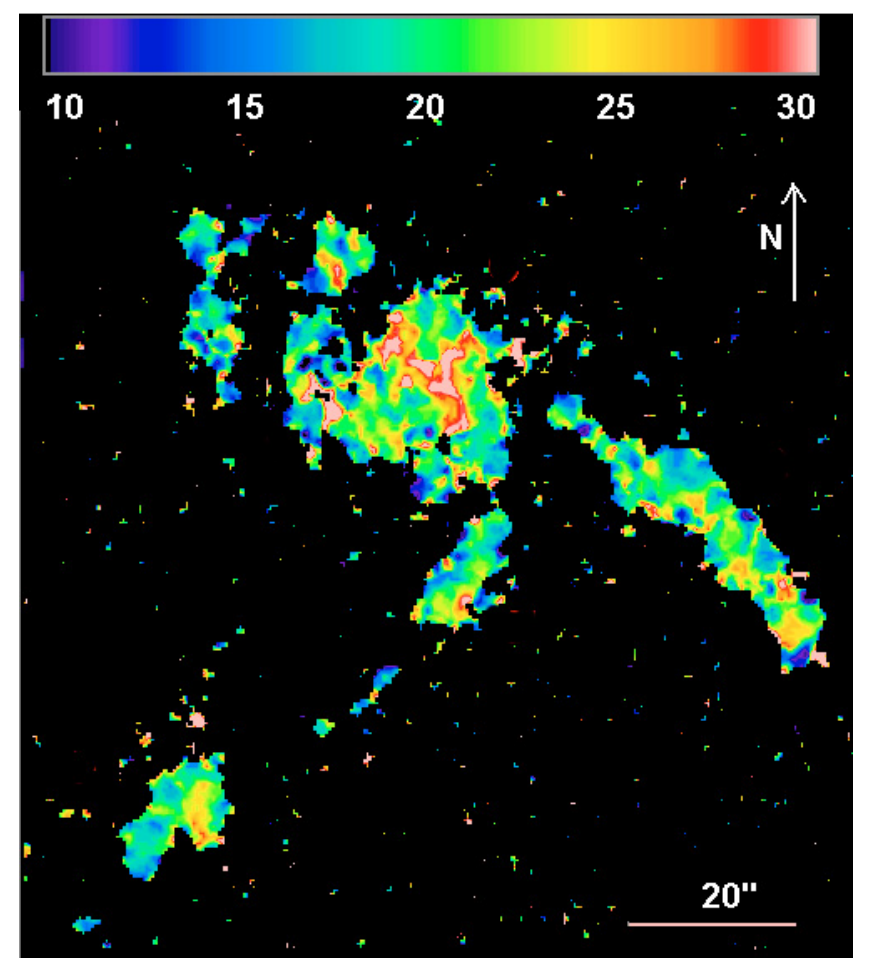

Fig. 8. Color $\mathrm{H} \alpha$ velocity dispersion of HCG 31. The scale is labeled in $\mathrm{km} \mathrm{s}^{-1}$.

velocity fields compare quite well, respecting the differences in resolution and extent. 
The velocity components of HCG 31 span a very narrow range of velocities, between 3950 and $4200 \mathrm{~km} \mathrm{~s}^{-1}$. Galaxy B alone spans almost this entire range. There is, however, no simple overall dynamic pattern for the group as a whole. The lowest velocities are found towards the South. The highest velocities occur in the SW of galaxy B but also along the NE tidal tail of galaxy A. The velocities do not display a jump through the region of junction between galaxies $\mathrm{A}$ and $\mathrm{C}$, at least not a large jump such as that observed, e.g. between galaxies $\mathrm{C}$ and $\mathrm{B}$, as expected.

Galaxy B exhibits a solid-body velocity field and RC. Significant non-circular motions are observed along the minor axis of the velocity field. Galaxy B is kinematically distinct from galaxies $\mathrm{A}+\mathrm{C}$ and is counter-rotating with respect to it. Nevertheless, a faint optical bridge which consists of a chain of $\mathrm{HII}$ regions, links galaxy $\mathrm{B}$ to galaxies $\mathrm{A}+\mathrm{C}$. This bridge is visible in the HST composite image from Johnson \& Conti (2000) and is also visible in our $\mathrm{H} \alpha$ image and $\mathrm{H} \alpha$ velocity field. It may be constituted of tidal material, as its radial velocity is the same as the radial velocity of the adjacent material belonging to galaxies A+C. Given its broadband morphology (Rubin et al. 1990; Iglesias-Páramo \& Vílchez 1997), it is most likely a dwarf spiral or an irregular seen nearly edge-on.

The $\mathrm{H} \alpha$ emission is not strong enough to be detected for the whole extent of the southern tidal arm. Our $\mathrm{H} \alpha$ images, as well as the HST and ground-based images from WIYN of HCG 31 (from Johnson \& Conti 2000), do not show continuous emission between galaxies $\mathrm{E}$ and $\mathrm{F}$ but instead a discrete emission centered on $\mathrm{H}$. The mean velocity of region $\mathrm{H}$ is $\sim 100 \mathrm{~km} \mathrm{~s}^{-1}$ higher than the mean velocity of object $\mathrm{F}$. The VLA integrated-intensity map of the HI emission (Williams et al. 1991; Verdes-Montenegro et al. 2005) clearly shows high quantities of neutral hydrogen all along the southern tail and extending to galaxy $\mathrm{G}$.

The lambda maps (Fig. 7) have been corrected from the FSR jumps. They show that the lowest velocity of the group ranges from the southern end of the southern tail to the overlapping $\mathrm{A}+\mathrm{C}$ regions. The highest velocities of the group are found in galaxy B, but A1 and A2 also display high velocities. The most interesting result from this map is that the overlapping region of galaxies $\mathrm{A}$ and $\mathrm{C}$, which displays a narrow range of velocities with amplitude $\sim 100 \mathrm{~km} \mathrm{~s}^{-1}$ ( from $\sim 3960$ to $\sim 4060 \mathrm{~km} \mathrm{~s}^{-1}$ ), also completely overlaps in velocity space, displaying a progressive superposition of the two disks. Galaxy A (C) dominates the highest (lowest) velocities of the $\mathrm{A}+\mathrm{C}$ system, and the large overlapping region (both position and velocity phase spaces) between both galaxies displays almost comparable flux levels with velocities within a range of $\sim 100 \mathrm{~km} \mathrm{~s}^{-1}$. This argues in favor of two disks approximately in the same plane in a pre-merging phase rather than a chance alignment of two components, one behind the other. If two disks experience a prograde encounter with almost parallel spins, a new disk will be formed. The formation of a new disk is reinforced when the star formation is strong, due to energy transfer, which is the case for HCG 31. Then, during the merging period, it may be difficult to find signatures of the two distinct disks in a system where collisions and/or strong tidal encounters have occurred. Nevertheless, as we are still able to separate the two components belonging to the two different disks in $\mathrm{HCG} 31 \mathrm{~A}+\mathrm{C}$, we are observing the pre-merging phase. These two disks can clearly be identified in the color image published in Fig. 2 from Johnson et al. (1999) where the red galaxy is $\mathrm{A}$ and the blue galaxy is $\mathrm{C}$.

The $\mathrm{H} \alpha$ velocity dispersion map is displayed in Fig. 8. The velocity dispersions have been computed from the HR data set,
Table 2. Kinematic Properties of the HCG 31 members.

\begin{tabular}{|c|c|c|c|c|c|}
\hline Name & \multicolumn{2}{|c|}{$\begin{array}{l}\text { Position Angle } \\
\text { (Degree) }\end{array}$} & \multicolumn{2}{|c|}{$\begin{array}{l}\text { Inclination } \\
\text { (Degree) }\end{array}$} & $\begin{array}{l}\text { Rotation Velocity } \\
\left(\mathrm{km} \mathrm{s}^{-1}\right)\end{array}$ \\
\hline$\overline{\text { HCG } 31}$ & $\begin{array}{l}\text { Velocity } \\
\text { map }\end{array}$ & $\begin{array}{l}\text { Cont. } \\
\text { map }\end{array}$ & $\begin{array}{l}\text { Velocity } \\
\text { map }\end{array}$ & $\begin{array}{l}\text { Cont. } \\
\text { map }\end{array}$ & $\begin{array}{l}\text { Approaching / Receding } \\
\text { side }\end{array}$ \\
\hline $\mathrm{A}+\mathrm{C}$ & $130 \pm 3$ & - & $52 \pm 5$ & - & $-60 /+280$ \\
\hline B & $45 \pm 3$ & $40 \pm 10$ & $60 \pm 5$ & $78 \pm 7$ & $-120 /+150$ \\
\hline $\mathrm{E}$ & $141 \pm 5$ & $145 \pm 5$ & $51 \pm 5$ & $62 \pm 5$ & $-10 /+45^{*}$ \\
\hline $\mathrm{F}$ & $138 \pm 5$ & $132 \pm 5$ & $55 \pm 5$ & $57 \pm 5$ & $0 /+10$ \\
\hline
\end{tabular}

* Sinusoidal shape.

deconvolved from the instrument point-spread function. Since the profiles can often be decomposed in several components, this map indicates the average width of the multiple profiles if any - rather than the width of any given individual component. The most important features in Fig. 8 are the broad profiles (larger than $30 \mathrm{~km} \mathrm{~s}^{-1}$ ) observed in the overlapping regions between galaxies $\mathrm{A}$ and $\mathrm{C}$, mapping the interaction between both galaxies and the subsequent starburst region. Indeed, these broad profiles indicate the presence of multiple components due to the merger. This is explained in detail in Sect. 5.2

\subsection{Description of the line-of-sight velocity plots and rotation curves of the HCG 31 members}

The kinematic properties of the members of the HCG 31 group are summarized in Table 2 and the line-of-sight heliocentric velocities (LOSVs) and RCs are presented in Fig. 9. The results presented in this section are based on LOSVs constructed by setting the center for each object on the location of the strongest stellar continuum for each object (the peaks in the continuum images). This choice usually implies that the LOSVs are quite asymmetric, and indeed both sides of the RC do not match. However, these asymmetries can be reduced, in most cases, if the center is set to be the kinematic center, instead. This indicates that the photometric and kinematic centers are decoupled.

The RCs were built by taking all the points inside a cone of semi-angle (hereafter $\mathrm{S}$ ) equal to 15 to $30^{\circ}$ around the kinematical major axis, depending on the object (see below). Kinematical parameters for the different RCs are presented. The inclination and the position angles are derived from the velocity map by minimizing the least-square deviation of the rotational velocity within the successive concentric crowns in the plane of the galaxy. The position angles used for the RCs are indicated as white segments in Fig. 1. The centers are indicated by black crosses in the same figure. These have been chosen to yield a $\mathrm{RC}$ with a shape as symmetric as possible and with matching of both sides of the curves, in the solid body regions.

Rotation curve of $\mathbf{B}\left(\mathrm{PA}=45 \pm 3^{\circ}, i=60 \pm 5^{\circ}, S=30^{\circ}\right.$, Fig. 9a). Regardless of the choice of kinematic parameters (position of the kinematic center, PA, i), both sides of the RC do not match and the RC shows a solid body behavior. In Fig. 9a the origin was set on the continuum center and the central $\mathrm{H} \alpha$ knot of $\mathrm{B}$. The disagreement between both sides is particularly obvious in the first five arcsec, and it may be the sign of a bar. The bridge between galaxies A and B is visible on the receding side of the RC beyond 20 arcsec.

Rotation curve of $\mathbf{E}\left(\mathrm{PA}=141 \pm 3^{\circ}, i=51 \pm 3^{\circ}, S=30^{\circ}\right.$, Fig. 9b). Regardless of the choice of parameters, the RC is asymmetric. If both sides of the $\mathrm{RC}$ match in the central regions (the first 2 arcsec), they show a strong disagreement in the external 

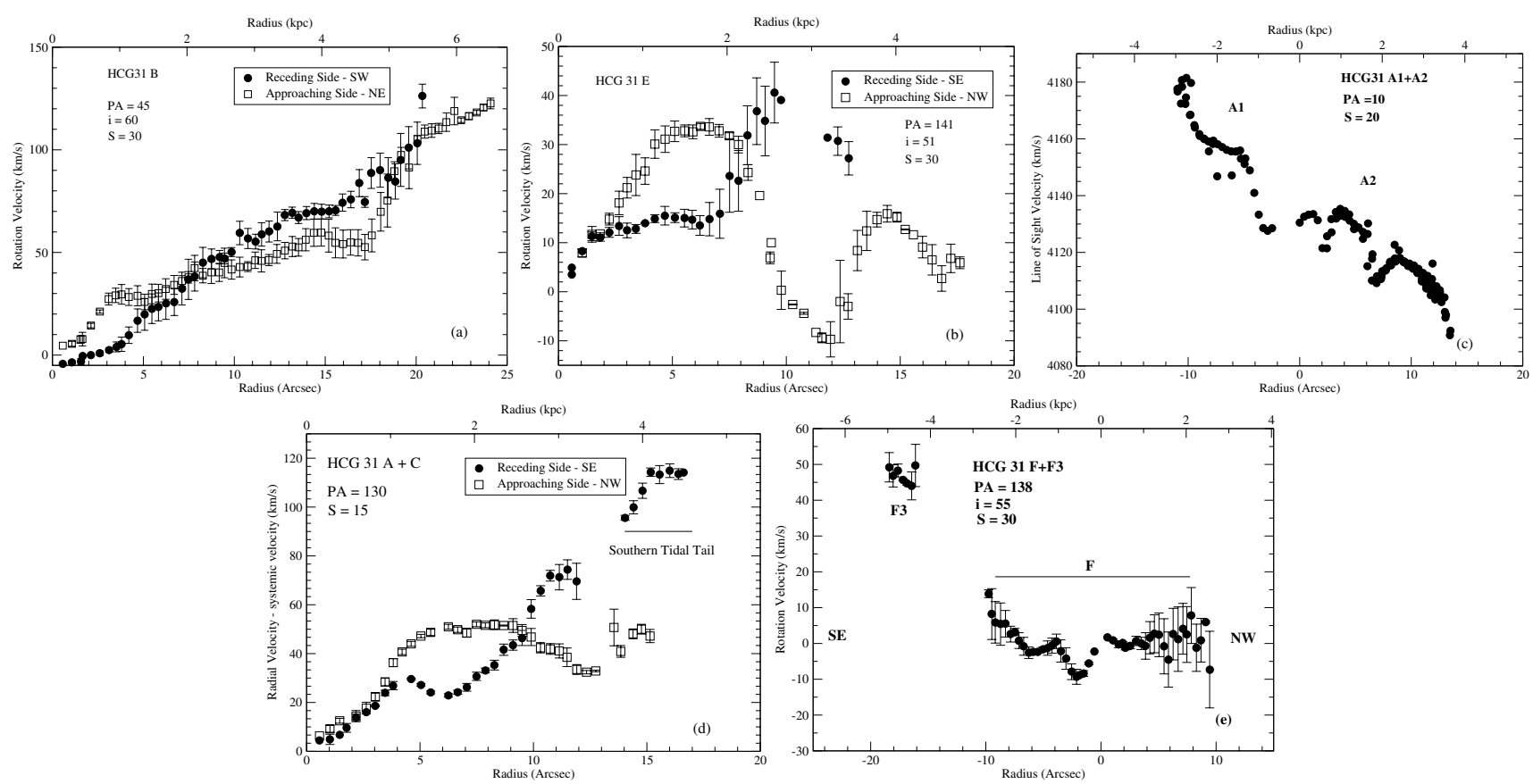

Fig. 9. a) Rotation curves of HCG 31 B. The origin of the radius corresponds to the central $\mathrm{H} \alpha$ knot of B. b) Rotation curves of object E, where the origin of the radius was displaced towards region E1. c) Position velocity diagram of A1 and A2. The center has been taken between A1 and A2. d) Rotation curve of the main velocity component of system A+C. e) Rotation curve of regions $\mathrm{F}$. The center has been taken between regions F1 and F2. See Fig. 1 for the location of the centers (black crosses) and orientations (white lines) of the slits used to extract the rotation curves shown above. " $\mathrm{S}$ " is the semi-angle used to delimitate a cone around the position angle in which the rotation curve has been calculated; "i" is the inclination and "PA" is the position angle of the major axis of the galaxy.

regions. In order to minimize the discrepancy between both sides, the center should be displaced in the direction of E1, along the line that joins the two regions, but then the agreement in the central region is lost.

Position-velocity plot through A1+A2 $\left(\mathrm{PA}=10^{\circ}, S=25^{\circ}\right.$, Fig. 9c). The position-velocity plot along a non linear is slit to match the curvature of the line joining the centers of A1 and A2. A velocity gradient of $90 \mathrm{~km} \mathrm{~s}^{-1}$ is observed along $\sim 7 \mathrm{kpc}$ ( 24 arcsec) through A1 and A2.

Position-velocity plot through A+C (PA $=130^{\circ}, S=15^{\circ}$, Fig. 9d). Both sides of the position-velocity plot do not match except in the first four arcsec. Then, the approaching side is flat while the receding side is increasing. The receding region of the plot between 14 and 17 arcsec, with highest velocities, belongs to the southern part of the tidal tail and may be due to streaming motions. In contrast, streaming motions are not observed along the northern part.

Rotation curve of $\mathbf{F}\left(\mathrm{PA}=138^{\circ}, i=55^{\circ}, S=30^{\circ}\right.$, Fig. 9e). The RC is flat through F1 and F2; the velocities are $40 \mathrm{~km} \mathrm{~s}^{-1}$ higher for F3, the most southern blob of region $\mathrm{F}$.

\section{Comparison with previous works}

We present in Appendix A a detailed comparison between our results (FP, for Fabry-Perot data) and measurements available in the literature (LS for long-slit data) by Rubin et al. (1990), Nishiura et al. (2000), López-Sánchez et al. (2004), as well as FP data from Richer et al. (2003) and HI data from Verdes-Montenegro et al. (2005). The spectral and spatial resolution for the various optical and HI data available in the literature for HCG 31 are summarized in Table 3. Our HR data set have the highest spatial and spectral resolution and allowed detection of HII regions at larger radii for each component. To
Table 3. Comparison of the resolutions for the different $\mathrm{H} \alpha$ and $\mathrm{HI}$ studies of HCG 31 .

\begin{tabular}{|c|c|c|}
\hline & \multicolumn{2}{|c|}{ "Spectral resolution Spatial resolution } \\
\hline & $\mathrm{km} \mathrm{s}^{-1}$ & $\operatorname{arcsec}$ \\
\hline Rubin et al. (1990) & 112 & 0.7 \\
\hline Nishiura et al. (2000) & 157 & 1.75 \\
\hline Richer et al. (2003) & 38.4 & 0.59 \\
\hline López-Sánchez et al. (2004) & 54 & 0.20 \\
\hline Verdes-Montenegro et al. (2005) & 10.6 & $15.8 \times 14.5$ \\
\hline Present Paper (2 data sets) & $16 / 3.2$ & $0.91 / 0.405$ \\
\hline
\end{tabular}

make the comparison possible between FP maps and slit spectroscopy, LOSVs and RCs were obtained within a mimicked slit through the FP data.

Our FP data generally agree well with all LS data, as well with other FP data. Some small discrepancies exist with the LS data with Rubin et al. (1990) and HI data from Verdes-Montenegro et al. (2005). Further details about these discrepancies are given in Appendix A.

\section{Discussion}

\subsection{Dwarf galaxy candidates in the tidal debris of HCG 31?}

Tidal dwarf galaxies (TDGs) originate within tidal tails resulting from strong interactions between two disk galaxies (Duc et al. 2004; Bournaud \& Duc 2006). Interacting compact groups may constitute an ideal environment to produce tidal dwarf galaxies.

The kinematics of tidal tails in colliding galaxies has already been studied via Fabry-Perot observations of the $\mathrm{H} \alpha$ emission (Bournaud et al. 2004). Large-scale velocity gradients due to streaming motions along the tails and small-scale motions related to the internal dynamics of giant HII regions within the 
tails have been used to show that, in several cases, genuine accumulations of matter were formed. Some of these structures are real and are kinematically decoupled from the rest of the tail. Such massive and self-gravitating objects are the progenitors of tidal dwarf galaxies.

Hunsberger et al. (1996) carried out a search for TDGs in the surroundings of Hickson compact group galaxies (Hickson et al. 1992) using $R$-band photometry, and they presented a sample of 47 candidates. Six of them belonged to HCG 31: three in the NW tidal tail of galaxy A (A1 and A2), one in the northern tail of system $\mathrm{A}+\mathrm{C}$, and two in the southern tail (one of which is object $\mathrm{E}$ ). A search for star-forming objects belonging to tidal tails has been carried out in a sample of deep $\mathrm{H} \alpha$ images of Iglesias-Páramo \& Vílchez (2001). For HCG 31 they found three objects that correspond to the three knots of $F$.

As shown in the previous sections, the rotation and velocity dispersion of $\mathrm{A} 1$ and $\mathrm{F}$ are very small, so these giant complexes are not massive enough to be gravitationally bound. They are, most probably, integral components of the $\mathrm{A}+\mathrm{C}$ complex and not separate entities, except, of course, if these lie and rotate in an almost face-on plane. In such a case, one does not expect to observe radial velocities and rotational velocities. If we assume that $\mathrm{F}$ has a disk shape (instead of a spheroidal one), based on the fact that the profiles are not broader for $F$ than on average elsewhere in the group, F may indeed lie face-on and may be supported gravitationally by rotation rather than by velocity dispersion. Nevertheless, based upon the morphology of F, which is not a round object, we may estimate that this region does not lie in a plane parallel to the plane of the sky. This does not apply to $\mathrm{A} 1$, which seems to be a round object and could present a flat $\mathrm{RC}$ due to projection effects.

Unlike objects A1 and F, objects A2 and E have velocity gradients with amplitudes of $\sim 25 \mathrm{~km} \mathrm{~s}^{-1}$ and they counter-rotate with respect to the main body A+C. Amram et al. (2004) computed from the mass of the central object and the distances and velocities of these components that they will fall back onto the parent galaxy. In fact, the counter-rotation measured in the velocity fields of A2 and E indeed indicates that the material is already returning to the main body.

Another piece of information that may make the TDG candidates in HCG 31 suspicious is their lack of CO emission (Yun et al. 1997). Molecular gas is abundant in the TDGs found in other systems, in quantities of up to a few $10^{8} M_{\odot}$ (Braine et al. 2001). Molecular gas should be present in large quantities in HCG 31 to allow the formation of a new generation of stars ionizing the huge HII complexes observed. The $\mathrm{CO}$ emission may still be present under the detection limit. New CO observations are needed, particularly including the northern part of the group around the TDG candidate "R" (Mendes de Oliveira et al. 2006).

In this paper we show that objects $\mathrm{A} 1, \mathrm{~A} 2, \mathrm{E}$, and $\mathrm{F}$ are probaldy not dwarf galaxies but tidal debris. A1 and A2 are not as strong star-forming objects as those in the southern tail $(\mathrm{E}$ and $\mathrm{F})$. The future of this tidal material is uncertain. Nevertheless, $(i)$ the low-velocity dispersion of these fragments within the group; (ii) their low mass; and (iii) their relatively small projected distances from the progenitor indicate they should not escape the potential well of the merger and may fall into the merger system. Object $F$ will live longer than objects $E$ and $\mathrm{H}$ because it is formed near the tip of the tail and should typically survive $\sim 2$ Gyrs or more, and possibly become a satellite galaxy of tidal origin. In contrast, objects formed at smaller radii (E, H) fall back and/or are disrupted in 500 Myrs (Bournaud \& Duc 2006). Another hypothesis that we favor, taking into account the large quantity of $\mathrm{HI}$ that the group possesses and the early dynamical stage of merging of the group, is that at least $\mathrm{F}$ is a TDG in the process of formation. Indeed, F may become more massive by continuous gas accretion and may, at the same time, escape from the progenitor, pushed away by the streaming motions within the tidal tails in development.

\subsection{The nature of the $A+C$ system: the pre-merging scenario}

The Hickson group HCG 31 has one of the lowest-velocity dispersions among all groups in Hickson's catalogue. Furthermore galaxies $\mathrm{A}$ and $\mathrm{C}$ have almost the same radial velocity. Several pieces of evidence indicate that the progenitors of the merger $\mathrm{A}+\mathrm{C}$ are late-type, gas-rich, large magellanic-type irregular galaxies: $(i)$ the emission line ratios and abundances given by Rubin et al. (1990) and López-Sánchez et al. (2004); (ii) the amount of HI gas associated with the group; (iii) the small velocity gradients within each galaxy; (iv) the photometric properties of the starburst (Iglesias-Páramo \& Vílchez 1997); (v) and the small size of the present entire complex.

Verdes-Montenegro et al. (2001) propose an evolutionary sequence of compact groups, based on the HI distribution in these systems. Verdes-Montenegro et al. (2005) classify HCG 31 as a system in an intermediate phase, in which multiple tidal tails form and a large amount of the atomic gas is found in the intragroup medium. In the same classification, HCG 92 (the Stephan's Quintet) is a much more evolved group than HCG 31, since it has all its HI in the intra-group medium. Surace (1998) classified interacting systems in five dynamical stages, first approach, first contact, pre-merger, merger, and old merger. HCG 31 clearly belongs to the class of pre-mergers, described by Surace as "two nuclei with well-developed tidal tails and bridges". The conditions for a merger to occur, i.e., the signs that a merger will occur in HCG 31, are:

- The overlapping components throughout the system. Indeed, as the extinction increases with the star formation rate (e.g. Buat \& Xu 1996), if one galaxy were behind the other, the background component would be highly extincted. Being able to clearly see the two components with similar intensities indicates that they lie almost in the same layer and they are mixed.

- The wider velocity dispersion profiles observed in the interface between galaxies A and C. As the disks are not transparent, as explained above, the wider velocity dispersion component could mean that the two systems still have different but similar velocities and are not totally relaxed. Close pairs with overlapping disks that have starbursts in their overlapping regions are either advanced mergers or severely colliding systems (e.g. Xu et al. 2000). In addition, Xu et al. (2000) find that for one out of the three advanced-merger systems in their sample, the starburst in the overlapping region is stronger than the central starburst, which is exactly the case for HCG 31. These arguments are strong indicators that the galaxies are either severely colliding or merging.

- The kinematic peculiarities visible in the velocity fields, as can be seen in the velocity curves shown in Fig. 9. No signature is seen of distinct rotating disks.

- The presence of tidal tails. This indicates that the interacting galaxies have had at least one earlier passage, which suggests that the galaxies are in a bound orbit (Bournaud \& Duc 2006). Stripping of gas is ubiquitous, in agreement with the finding from Verdes-Montenegro et al. (2005) that $60 \%$ of the HI is located in the tidal tails. This clearly indicates that the galaxies have already experienced tidal interaction. 
- The very small separation between galaxies A and C. In contrast to the previous statements above, this alone is a necessary but not sufficient condition for the merging given that the tangential velocities are not known. However, taken together with the above condition (presence of tidal tails and TDGs), it becomes sufficient to attest to the physical proximity of both galaxies.

Numerical simulations of merging disks indicate that the nuclear separation between the two galaxies in the system is not simply a monotonically decreasing function of time. The system often goes through a pre-merger phase characterized by two distinct galaxies with well-developed tidal tails and bridges as a result of the first close encounter (Barnes \& Hernquist 1992, 1996; Mihos \& Hernquist 1996; Springel 2000). Thus, the presence of tails clearly indicates that the system does not have an open relative trajectory (parabolic or hyperbolic). Unbound orbits (corresponding to approaching velocities higher than $\sim 500 \mathrm{~km} \mathrm{~s}^{-1}$ ) are inefficient in producing tidal tails (Binney \& Tremaine 1987; Bournaud \& Duc 2006) and hence do not form TDGs. For unbound orbits like the ones observed in face-on collisions (e.g. cartwheel-type galaxies or NGC 5291-type systems), recycled objects may appear in the expanding ring around the target, but these recycled objects are not TDGs (Bournaud \& Duc 2006). Furthermore, the average dynamical time for the double-nucleus galaxies to coalesce into one single nucleus owing to dynamical friction is less than $1.2 \times 10^{8}$ yrs (Colina et al. 2001).

The detailed fitting of the high-resolution data set, showing double velocity profiles in the central object $A+C$, reveals the several kinematical disturbances described in the previous sections. In addition, the observed morphological disturbances, the presence of well-developed tails and gaseous condensations in the northern and southern tails of HCG 31, are strong indicators that galaxies $\mathrm{A}+\mathrm{C}$ are observed after the second moment of closest approach. If it were around the first approach, we would not expect the kinematic signatures we observed. In addition, with the small observed nucleus separation between $A$ and $C(2 \mathrm{kpc})$, we would expect smaller tidal tails, instead of the well- developed tails observed and no gaseous condensation in the tails (e.g. Hibbard 1995; Mihos \& Hernquist 1996; Springel 2000). We do not support the suggestion by Richer et al. (2004) that the southern tidal tail and the condensations are, instead, created by a close fly-by interaction with galaxy $\mathrm{G}$. Besides the arguments given above, we can add the fact that the velocity field of $\mathrm{G}$ is fairly undisturbed (Verdes-Montenegro et al. 2005). Both galaxies $\mathrm{G}$ and $\mathrm{Q}$ are embedded in a common HI envelope (Williams et al. 1991) in which optical tidal debris may be present, and they have radial velocities similar to those of the rest of the group (Mendes de Oliveira et al. 2006). They may therefore be involved in the interaction but not directly responsible for the creation of the southern tail.

Comparison between numerical simulations and the stellar (e.g. Johnson \& Conti 2000) and gaseous (Verdes-Montenegro et al. 2005) tidal tails clearly indicate a prograde encounter between galaxies $\mathrm{A}$ and $\mathrm{C}$. This configuration is confirmed by the general $\mathrm{H} \alpha$ velocity pattern of $\mathrm{A}+\mathrm{C}$ : the two disks in the process of merging still rotate with parallel spin axis, like a set of gear wheels. Indeed, from the velocity field, the spin axis cannot be anti-parallel. We note that prograde encounters and high star-formation rates favor the formation of a new disk.

The general pattern of the velocity field indicates that the inclinations of the pre-merging galaxies $\mathrm{A}$ and $\mathrm{C}$ were rather similar in the past and are still similar now. Indeed, most probably, violent relaxation processes have just started in this system, and the angular momentum has not yet been redistributed. As can be seen from the two separate HI clouds (Verdes-Montenegro et al. 2005), galaxies A and C still keep their individual HI disks.

All arguments above, which relate to the conditions itemized in this section motivated us to support the scenario in which the galaxies are in a pre-merger phase (Amram et al. 2004; Verdes-Montenegro et al. 2005). Two other scenarios have been put forward for $\mathrm{A}+\mathrm{C}$. Our data do not support the other two possible scenarios discussed in the literature i.e. that $\mathrm{A}+\mathrm{C}$ is neither a late stage merger nor a single galaxy.

\section{Summary and conclusions}

We have obtained new Fabry-Perot data, $\mathrm{H} \alpha$ images, velocity fields, and velocity dispersion maps, position-velocity diagrams and rotation curves of the Hickson compact group HCG 31. We described the internal kinematics of the group HCG 31 in detail. A comparison with previously published long-slit spectroscopy, Fabry-Perot and HI data show good agreement in the rotation curves of the different components. Our data nevertheless benefit from a much higher spectral and spatial resolution than the other Fabry-Perot data and allowed detection of HII regions at larger radii from each component.

This paper complements our previous work on the same group (Amram et al. 2004). Our main conclusions are that:

- Galaxies A and C still bear signs of the systematic rotation patterns of the individual systems. We bring new evidence that the $\mathrm{A}+\mathrm{C}$ system is in an early stage of merging due to the presence of double profiles of the $\mathrm{H} \alpha$ line in the region between $\mathrm{A}$ and $\mathrm{C}$. The velocity dispersion map shows widespread low-velocity dispersion throughout the group except in the interface where the merger between $\mathrm{A}+\mathrm{C}$ occurs. We conclude that $\mathrm{A}+\mathrm{C}$ is in a pre-merger phase and the encounter of the two objects was in a prograde orbit.

- The velocity curves of four candidates tidal dwarf galaxies (TDGs) show flat velocity patterns in two cases (A1 and F) and very small gradients (E and A2, amplitude $\sim 25 \mathrm{~km} \mathrm{~s}^{-1}$ ). Therefore these candidates are not TDGs but rather tidal debris that could fall back onto the larger galaxies. At least (F), one of them could be a TDG candidate in formation by continuous HI gas accretion.

Numerical simulations would be needed to constrain the parameters of the interaction of this compact group including all members, particularly (A, B, C, G, and Q), which may have contributed to the morphological and dynamical status of the group. If will be challenging to reproduce the morphological and kinematic features of the group observed in the gas component, as well as in the old and young stellar components.

Acknowledgements. The authors thank J.L. Gach and C. Carignan for helping during the observations and F. Bournaud and P.-A. Duc for interesting discussions. CMdO would like to acknowledge support from the Brazilian agencies FAPESP (projeto temático 01/07342-7), CNPq and CAPES. P.A. and C.B. would like to thank COFECUB for its financial support. HP wishes to thank the Scientific Council of the Observatoire Astronomique de Marseille Provence for financial support during his visit in January and February 2004. We made use of the Hyperleda database and the NASA/IPAC Extragalactic Database (NED). The latter is operated by the Jet Propulsion Laboratory, California Institute of Technology, under contract with NASA. The authors thank the anonymous referee who helped to improve the paper. 


\section{References}

Amram, P., Boulesteix, J., Georgelin, Y. M., et al. 1991, The Messenger, 64, 44 Amram, P., Le Coarer, E., Marcelin, M., et al. 1992, A\&AS, 94, 175

Amram, P., de Oliveira, C., Mendes, P. H., et al. 2004, ApJ, 612, L5

Amram, P., Plana, H., Mendes de Oliveira, C., Balkowski, C., \& Boulesteix, J. 2003, A\&A, 402, 865

Barnes, J. E., \& Hernquist, L. 1992, Nature, 360, 715

Barnes, J. E., \& Hernquist, L. 1996, ApJ, 471, 115

Binney, J., \& Tremaine, S. 1987, Galactic Dynamics (Princeton Series in Astrophysics), 455

Boulesteix, J. 2002, ADHOCw reduction Package (Publication of Observatoire de Marseille), http://www . oamp. fr/adhoc/

Bournaud, F., \& Duc, P.-A. 2006, A\&A, 456, 481

Bournaud, F., Duc, P.-A., Amram, P., Combes, F., \& Gach, J.-L. 2004, A\&A, 425,813

Braine, J., Duc, P.-A., Lisenfeld, U., et al. 2001, A\&A, 378, 51

Buat, V., \& Xu, C. 1996, A\&A, 306,61

Colina, L., Borne, K., Bushouse, H., et al. 2001, ApJ, 563, 546.

Duc, P.-A., Bournaud, F., \& Masset, F. 2004, A\&A, 427, 803

Gach, J.-L., Hernandez, O., Boulesteix, J., et al. 2002, PASP, 114, 1043

Hibbard, J. E. 1995, Ph.D. Thesis, Columbia University

Hickson, P. 1982, ApJ, 255, 382

Hickson, P., Mendes de Oliveira, C., Huchra, J. P., \& Palumbo, G. G. C. 1992, ApJ, 399, 353

Hunsberger, S. D., Charlton, J. C., \& Zaritsky, D. 1996, ApJ, 462, 50

Iglesias-Páramo, J., \& Vílchez, J. M. 1997, ApJ, 479, 190
Iglesias-Páramo, J., \& Vílchez, J. M. 2001, ApJ, 550, 204

Johnson, K. E., \& Conti, P. S. 2000, AJ, 119, 2146

Johnson, K. E., Vacca, W. D., Leitherer, C., Conti, P. S., \& Lipscy, S. J. 1999, AJ, 117, 1708

López-Sánchez, Á. R., Esteban, C., \& Rodríguez, M. 2004, ApJS, 153, 243

Mendes de Oliveira, C., \& Hickson, P. 1994, ApJ, 427, 684

Mendes de Oliveira, C., Amram, P., Plana, H., \& Balkowski, C. 2003, AJ, 126, 2835

Mendes de Oliveira, C., Temporin, S., Cypriano, E. S., et al. 2006, AJ, 132, 570

Mihos, J. C., \& Hernquist, L. 1996, ApJ, 464, 641

Nishiura, S., Shimada, M., Ohyama, Y., Murayama, T., \& Taniguchi, Y. 2000, AJ, 120, 169

Plana, H., Amram, P., Mendes de Oliveira, C., Balkowski, C., \& Boulesteix, J. 2003, AJ, 125, 1736

Richer, M. G., Georgiev, L., Rosado, M., et al. 2003, A\&A, 397, 99

Rubin, V. C., Hunter, D. A., \& Ford, W. K. Jr. 1990, ApJ, 365, 86

Springel, V. 2000, MNRAS, 312, 859

Sulentic, J. W. 1987, ApJ, 322, 605

Surace, J. A. 1998, Ph.D. Thesis, University of Hawaii

Verdes-Montenegro, L., Yun, M. S. Williams, B. A., et al. 2001, A\&A, 377, 812

Verdes-Montenegro, L., Del Olmo, A., Yun, M. S., \& Perea, J. 2005, A\&A, 430, 443

Williams, B. A., McMahon, P. M., \& van Gorkom, J. H. 1991, AJ, 101, 1957

Williams, B. A., Yun, M. S., \& Verdes-Montenegro, L. 2002, AJ, 123, 2417

Xu, C., Gao, Y., Mazzarella, J., et al. 2000, ApJ, 541, 644

Yun, M. S., Verdes-Montenegro, L., Del Olmo, A., \& Perea, J. 1997, ApJ, 475, L21 
P. Amram et al.: HCG 31, Online Material p 1

\section{Online Material}




\section{Appendix A: Comparison with previous works}

\section{A.1. Comparison between our Fabry-Perot data and Rubin et al. (1990) data}

We compared our data with those of Rubin et al. (1990) who presented LOSVs for A, B, C, and E from long-slit spectroscopy. The spectra obtained by those authors have a spectral resolution of $\leq 2.46 \AA \sim 112 \mathrm{~km} \mathrm{~s}^{-1}$ (for a slit width of 2 arcsec). We overplot the two data sets in Fig. A.1. In order to simulate a slit, we have taken an angular sector of $\pm 5^{\circ}$ throughout our data. Our main results are:

Long slit through $\mathbf{A}+\mathbf{B}\left(\mathrm{PA}=88^{\circ}\right.$, Fig. A.1a). Both data sets are apparently consistent. Nevertheless, if we strictly simulate a 2 arcsec-wide long slit, we are unable to reproduce the western part of the LOSV beyond 20 arcsec from the nucleus. A better match of the two curves is obtained, however, if we use a cone (instead of a slit) with an angular sector of $\pm 10^{\circ}$, given that this will then include regions of galaxy A which are $20 \operatorname{arcsec}$ to 35 arcsec away from the nucleus, measured in the Rubin et al. (1990) work.

Long slit through $\mathbf{C}+\mathbf{B}\left(\mathrm{PA}=40^{\circ}\right.$, Fig. A.1b). The SW part of the LOSV plot corresponds to B, while the NE part corresponds to $\mathrm{C}$. The agreement between the LS and the FP data for B is good. In contrast, for A, if we strictly simulate a 2 arcsec-wide long slit, we miss the NE bridge of B (connecting $\mathrm{B}$ to $\mathrm{C}$ ) and the LS and FP curves present a disagreement of $\sim 50 \mathrm{~km} \mathrm{~s}^{-1}$. In order to include the bridge located between 20 arcsec and 30 arcsec NE from the nucleus of B, using the same PA, we have to simulate a cone with an angular sector of $\pm 10^{\circ}$. Using a cone instead of a slit, the FP LOSV has globally the same trend, but the disagreement between the LS and the FP data remains.

Long slit through $\mathbf{C}\left(\mathrm{PA}=20^{\circ}\right.$, Fig. A.1c). The LOSV is very irregular. The global shapes of both data sets are roughly similar, but there is a shift of $\sim+20 \mathrm{~km} \mathrm{~s}^{-1}$ between them.

Long slit through $\mathbf{F}+\mathbf{E}+\mathbf{B}\left(\mathrm{PA}=135^{\circ}\right.$, Fig. A.1d). The agreement between the LS and the FP data is acceptable to the SE of the nucleus of E, except for a shift of $\sim 10 \mathrm{~km} \mathrm{~s}^{-1}$. To the $\mathrm{NW}$ of the nucleus of $\mathrm{E}$, the shapes of the LOSVs disagree.

\section{A.2. Comparison between our Fabry-Perot data and Nishiura et al. (2000) data}

We compare our data with those of Nishiura et al. (2000) who presented RCs for A, B, and C from long-slit spectroscopy. We plot the two data sets using the same parameters and the center given by the continuum emission. To simulate the slit, we have taken a half angular sector of $\pm 5^{\circ}$. The main results are:

Long slit through A (PA $=80^{\circ}, i=62^{\circ}$, Fig. A.2a). Both data sets show consistent velocity amplitudes and coverages; nevertheless, the shape of the curves within the central \pm 10 arc$\sec ( \pm 2.8 \mathrm{kpc})$ disagrees by $\sim 20 \pm 5 \mathrm{~km} \mathrm{~s}^{-1}$.

Long slit through $\mathrm{B}\left(\mathrm{PA}=41^{\circ}, i=60^{\circ}\right.$, Fig. A.2b); the curves are in good agreement.

Long slit through $\mathbf{C}\left(\mathrm{PA}=41^{\circ}, i=70^{\circ}\right.$, Fig. A.2c). Despite the irregularities of the curves and the difference in sampling, the agreement is fairly good. The position angle corresponds to the optical orientation of the main body but does not match any specific orientation of the velocity field.

\section{A.3. Comparison between our Fabry-Perot data and Richer et al. (2003) data}

Richer et al. (2003) plotted their Fabry-Perot data using the Rubin et al. (1990) parameters but did not overplot the two data sets. Below we compare the Richer et al. (2003) data and ours, using the parameters given in Rubin et al. (1990).

Long slit through $\mathbf{A}\left(\mathrm{PA}=88^{\circ}\right.$, Fig. A.3a). Both data sets are consistent, although our FP data extends to larger radii.

Long slit through B (PA $=40^{\circ}$, Fig. A.3b). Both data sets are consistent between -15 and +15 arcsec $(\mathrm{SW}, \pm 4 \mathrm{kpc}$ ), but the spatial coverage of the Richer et al. data between the three main bright $\mathrm{H} \alpha$ knots is not complete. Furthermore, beyond -15 $\operatorname{arcsec}(\mathrm{NE})$, Richer et al. do not detect the bridge between B and $\mathrm{A}$.

Long slit through $\mathbf{C}\left(\mathrm{PA}=20^{\circ}\right.$, Fig. A.3c). The LOSV is roughly flat and irregular along $40 \mathrm{arcsec}(10 \mathrm{kpc})$. Our FP data are less scattered and more extended than Richer et al.'s.

\section{A.4. Comparison between our Fabry-Perot data and López-Sánchez et al. (2004) data}

We compare our data with those of López-Sánchez et al. (2004), who presented LOSVs for A, B, C, E, F and H from long-slit spectroscopy. To simulate the slit, we have taken a half angular sector of $\pm 5^{\circ}$. The main results are:

Long slit through A2+A+C+B $\left(\mathrm{PA}=61^{\circ}\right.$, Fig. A.4a). Both data sets are roughly consistent. For galaxy $\mathrm{B}$, the FP curve is smoother. For galaxy $\mathrm{C}$, the phase of the oscillations of the curve depends on the exact location of the center. HII regions in the northern internal tidal arm of A have been detected only in the FP data; the velocities for region A2 are consistent between the two studies.

Long slit through $\mathbf{F}+\mathbf{H}+\mathbf{E}+\mathbf{B}\left(\mathrm{PA}=128^{\circ}\right.$, Fig. A.4b). The four objects are not exactly aligned. To compare the LS and our FP data we have centered our simulated slit along H. This implies that the slit is shifted towards the SW of the major axis of $\mathrm{E}$ and $\mathrm{F}$, so we miss the central regions of $\mathrm{E}$ and F. Velocity through object F, H, E, and B are in good agreement between both data sets. Two differences could nevertheless be pointed out: our FP data reach an HII complex SE of F (identified with a rather high signal-to-noise ratio), labeled F3, and the velocity gradient through $\mathrm{H}$ is $\sim 50$ and $\sim 30 \mathrm{~km} \mathrm{~s}^{-1}$ if measured from the FP and the LS data, respectively. The LOSV through $\mathrm{H} 1$ and $\mathrm{H} 2$ shows a velocity continuity with the receding side of $\mathrm{E}$. A velocity gradient of $50 \mathrm{~km} \mathrm{~s}^{-1}$ is observed along an extension of $13 \operatorname{arcsec}(5 \mathrm{kpc})$. This could be the signature of a massive clump with a dynamical mass of $M \sim 3 \times 10^{8} M_{\odot}$ or, alternatively, the signature of streaming motions along the southern tidal tail. Beyond region $\mathrm{H} 1$, the velocity drops by $100 \mathrm{~km} \mathrm{~s}^{-1}$ to object $\mathrm{F}$, which has a very flat $\mathrm{RC}$.

Long slit through $\mathbf{F}+\mathbf{H}+\mathbf{E}+\mathbf{B}\left(\mathrm{PA}=133^{\circ}\right.$, Fig. A.4c $)$. In order to get the central regions of $\mathrm{F}$ and $\mathrm{E}$, the $\mathrm{PA}$ of the simulated slit had to be slightly rotated with respect to that used by the authors. In both data sets (LS and our FP), region E displays a velocity gradient of $\sim 55 \mathrm{~km} \mathrm{~s}^{-1}$, along $15 \operatorname{arcsec}(4 \mathrm{kpc})$, giving a velocity gradient of $\sim 12.5 \mathrm{~km} \mathrm{~s}^{-1} \mathrm{kpc}^{-1}$. Region $\mathrm{H}$ (split in two regions $\mathrm{H}_{1}$ and $\mathrm{H}_{2}$ ) shows a velocity gradient of $\sim 60 \mathrm{~km} \mathrm{~s}^{-1}$ along $15 \operatorname{arcsec}(4 \mathrm{kpc}$ ), giving a velocity gradient of $\sim 15 \mathrm{~km} \mathrm{~s}^{-1} \mathrm{kpc}^{-1}$. Surprisingly, region $\mathrm{H}$ does not appear in the LS plot. Region F exhibits a very small velocity gradient along its $25 \operatorname{arcsec}(\sim 4 \mathrm{kpc})$. 

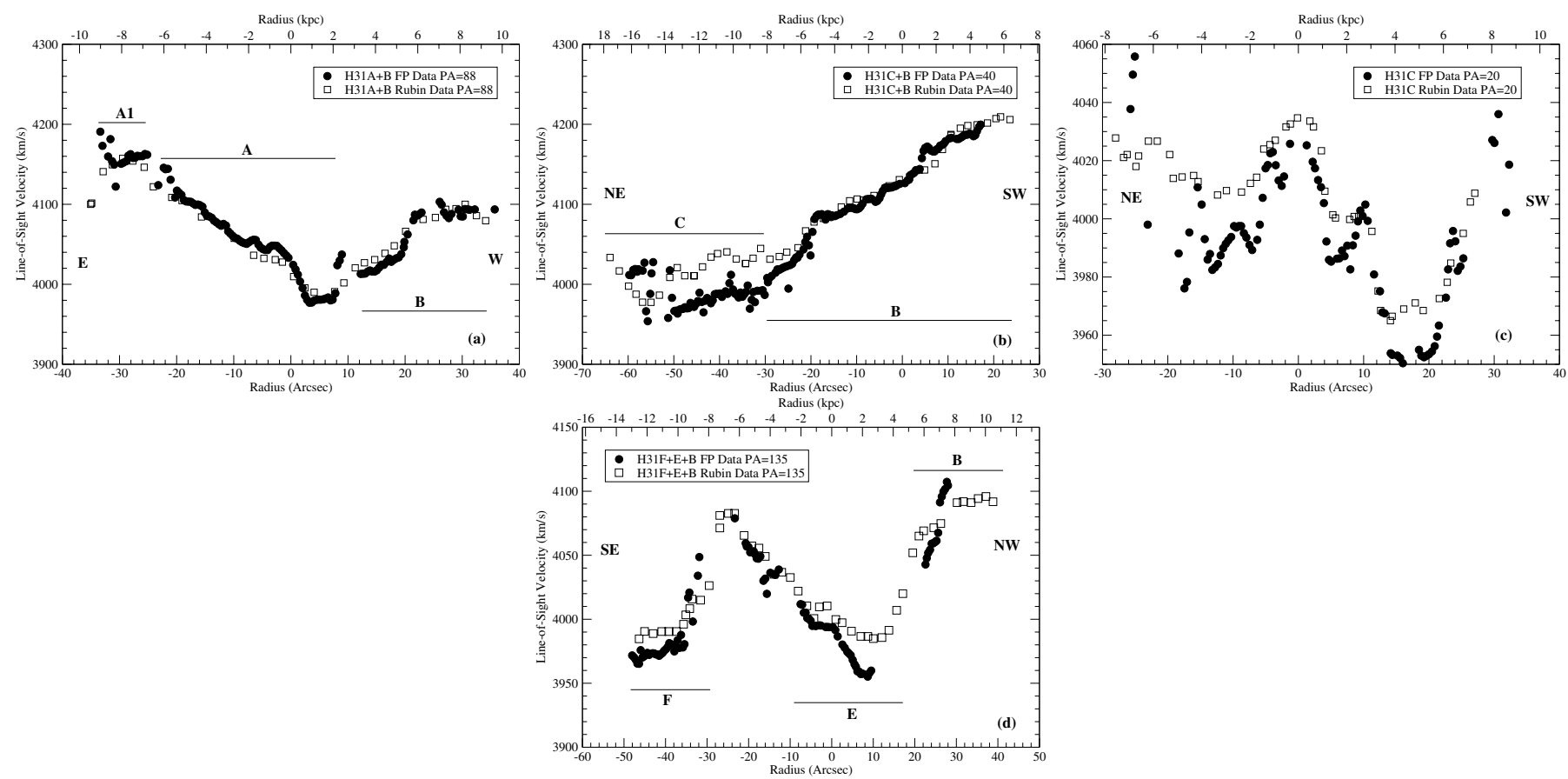

Fig. A.1. Comparison between long-slit data by Rubin et al. (1990) and the Fabry-Perot data made using line-of-sight velocity plots simulating a slit. a) Through HCG $31 \mathrm{~A}+\mathrm{B}$. The origin of the radius corresponds to the continuum center of A. b) Through HCG $31 \mathrm{C}+\mathrm{B}$. The origin of the radius corresponds to the central $\mathrm{H} \alpha$ knot of B. c) Through HCG $31 \mathrm{C}$. The origin corresponds to 2.5 arcsec North of the brighter knot of C. d) Through HCG $31 \mathrm{~F}+\mathrm{E}+\mathrm{B}$. d) The origin the of radius corresponds to the emission knot of region E2.
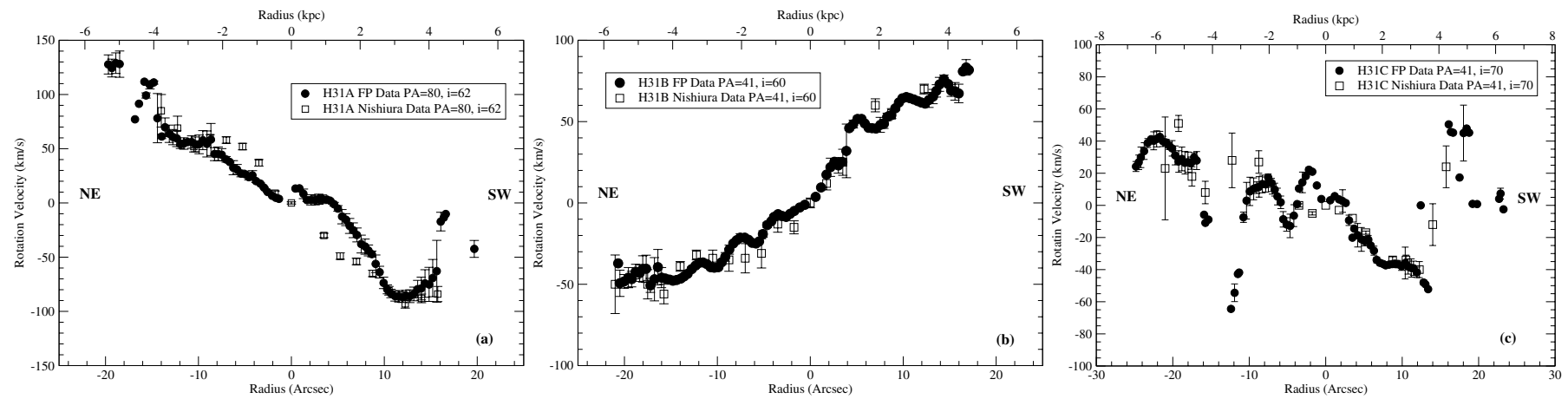

Fig. A.2. Comparison between long-slit data by Nishiura et al. (2000) and Fabry-Perot data made using rotation curves simulating a slit. a) Through HCG 31 A. The origin of the radius corresponds to a region 7 arcsec East of the brightest knot of $\mathbf{C} \mathbf{b})$ Through HCG $31 \mathrm{~B}$. The origin of the radius corresponds to the central $\mathrm{H} \alpha$ knot of B. c) Through HCG $31 \mathrm{C}$. The origin of the radius corresponds to a region 3 arcsec North of the brightest knot of C.
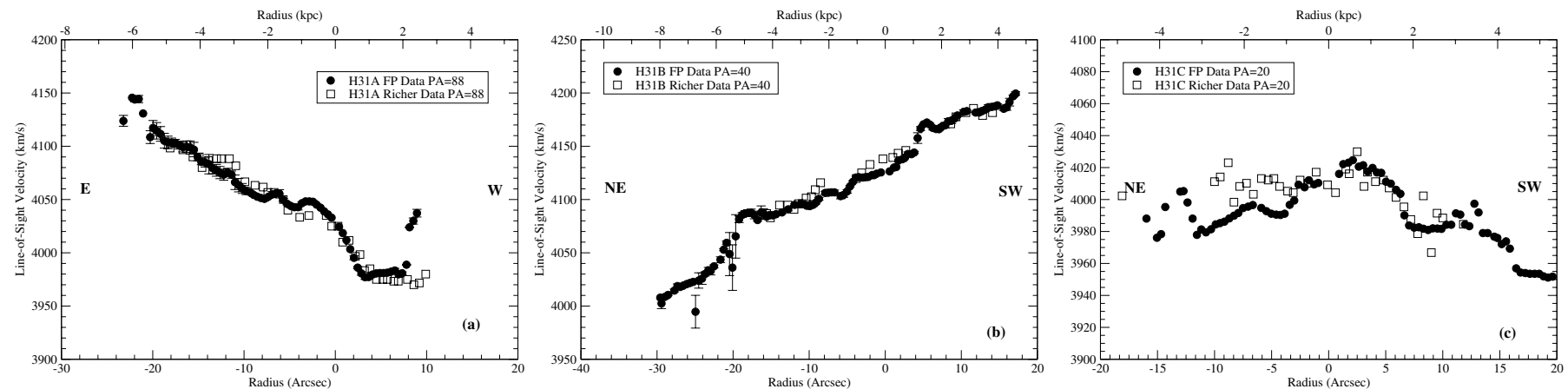

Fig. A.3. Comparison between long-slit data by Richer et al. (2003) and Fabry-Perot data made using line-of-sight Velocity plots simulating a slit. a) Through HCG $31 \mathrm{~A}$. The origin of the radius corresponds to the brightest knot of C. b) Through HCG $31 \mathrm{~B}$. The origin of the radius corresponds to the central $\mathrm{H} \alpha$ knot of B. c) Through HCG $31 \mathrm{C}$. The origin of the radius corresponds to a region located 2.5 arcsec North of the brightest knot of C. 

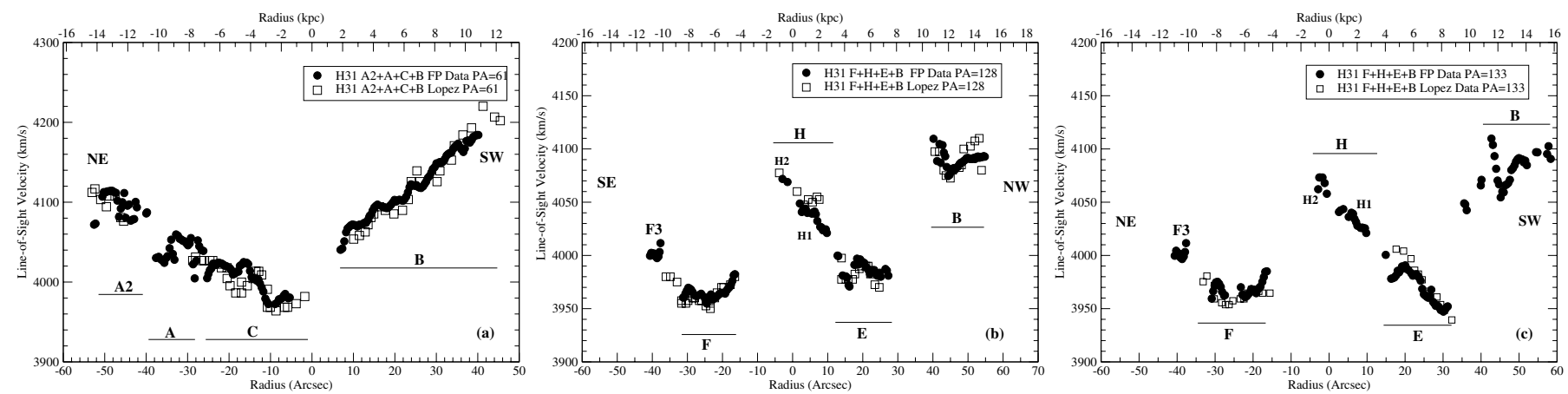

Fig. A.4. Comparison between long-slit data by López-Sánchez et al. (2004) and Fabry-Perot data made using line-of-sight velocity plots simulating a slit. a) Through HCG $31 \mathrm{~A}+\mathrm{B}+\mathrm{C}$. The origin of the radius corresponds to the middle distance between $\mathrm{C}$ and $\mathrm{B}$ continuum centers. b) Through HCG $31 \mathrm{~F}+\mathrm{H}+\mathrm{E}+\mathrm{B}$. The origin of the radius corresponds to middle distance between the $\mathrm{H} 1$ and $\mathrm{H} 2 \mathrm{knots}$. c) Same as b with a different position angle.
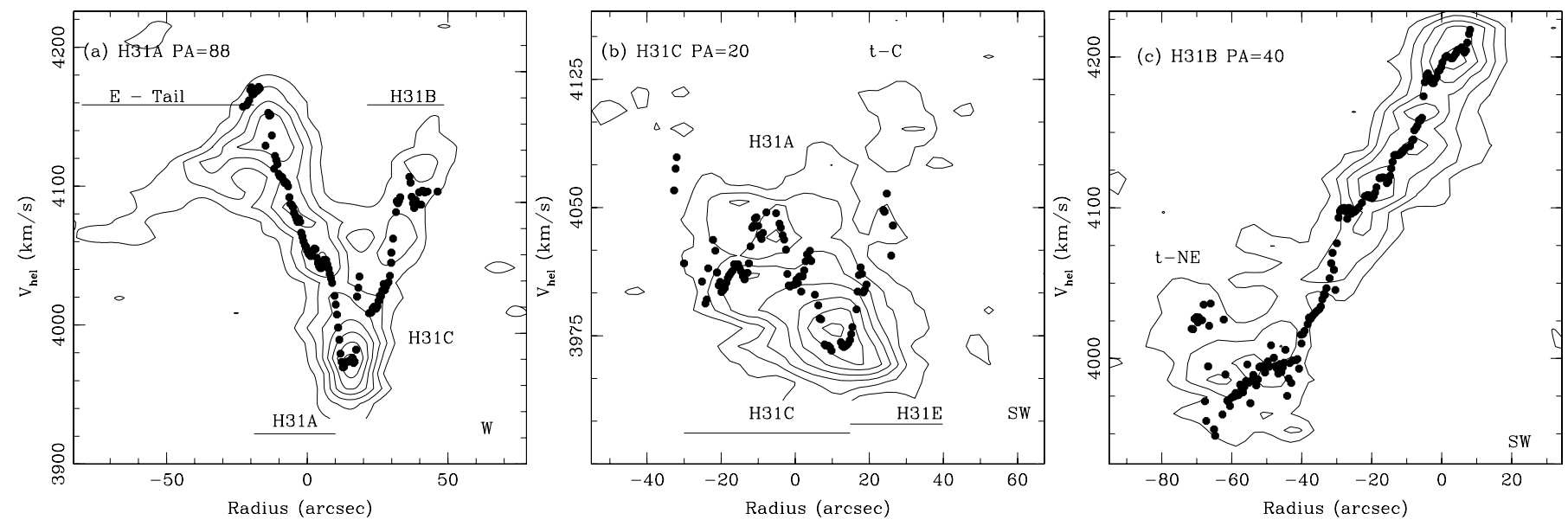

Fig. A.5. Comparison between HI position-velocity diagrams (isocontours) by Verdes-Montenegro et al. (2005) and high- resolution Fabry-Perot data (dots) made using line-of-sight velocity plots simulating a slit. a) Through HCG $31 \mathrm{~A}$, position angle PA $=88^{\circ}$. b) Through HCG $31 \mathrm{C}$, $\mathrm{PA}=20^{\circ}$. c) Through HCG $31 \mathrm{~B}, \mathrm{PA}=40^{\circ}$. The HI plotted levels are the same as in Verdes-Montenegro et al. (2005). The synthesized beam is $15.8 \times 14.5 \operatorname{arcsec}^{2}$.

\section{A.5. Comparison between our Fabry-Perot data and Verdes-Montenegro et al. (2005) data.}

We compare our data with those of Verdes-Montenegro et al. (2005), who presented LOSVs of HI features obtained along the position angle of 88,20 , and $40^{\circ}$ (Fig. A.5a-c).

Long slit through A (PA $=88^{\circ}$, Fig. A.5a). If we position our simulated slit 6 arcsec to the West of galaxy A, an excellent agreement is observed between the HI and our Fabry-Perot data, specifically in the overlapping region between galaxies $\mathrm{A}$ and $\mathrm{C}$.

Long slit through $\mathbf{C}\left(\mathrm{PA}=20^{\circ}\right.$, Fig. A.5b). Good agreement is achieved if the center of the slit is positioned 20 arcsec to the SW of galaxy A. The amplitude in velocity of the Fabry-Perot LOSV is larger than that of the HI LOSV.

Long slit through B (PA $=40^{\circ}$, Fig. A.5c). Good agreement is achieved if the center of the slit is positioned $10 \operatorname{arcsec} \mathrm{NE}$ of the center of galaxy A.

To optimize the superposition of the high-resolution FabryPerot data and the maximum intensities of the HI contours, keeping the same orientation for the simulated slit in the Fabry-Perot data as in the HI data, it was necessary to shift it slightly. This is due to the beam-smearing effect in the HI data (see Table 3). This effect may produce a shift in velocity if the velocity gradient is large compared to the beam size. This effect is related to possible intensity gradients within the beam size. Another consequence of the beam smearing is the lowering of the velocity amplitude in the HI data with respect to the Fabry-Perot data (as noted for instance for slit position PA $=40^{\circ}$ ). For the three different cuts presented here $\left(\mathrm{PA}=88,20\right.$ and $\left.40^{\circ}\right)$ as well as the other ones displayed in Verdes-Montenegro et al. (2005, but not presented here), if we take the beam smearing effect in the HI data into account, good agreement is observed between the two data sets. This means that the cold and the warm gas components trace the same potential well.

In conclusion, our FP data agree roughly with long slit-data (Rubin et al. 1990; Nishiura et al. 2000; Lopez-Sanchez et al. 2004), as well as with FP data from Richer et al. (2003) and HI data from Verdes-Montenegro et al. (2005). 